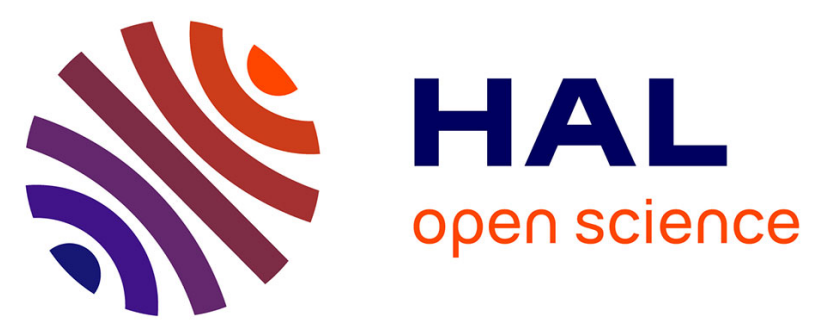

\title{
Quasi-Optimal Tone Reservation PAPR Reduction Algorithm for Next Generation Broadcasting Systems: A Performance/Complexity/Latency Tradeoff With Testbed Implementation
}

Sri Satish Krishna Chaitanya Bulusu, Matthieu Crussière, Jean-François

Hélard, Ralph Mounzer, Youssef Nasser, Olivier Rousset, Alain Untersee

\section{To cite this version:}

Sri Satish Krishna Chaitanya Bulusu, Matthieu Crussière, Jean-François Hélard, Ralph Mounzer, Youssef Nasser, et al.. Quasi-Optimal Tone Reservation PAPR Reduction Algorithm for Next Generation Broadcasting Systems: A Performance/Complexity/Latency Tradeoff With Testbed Implementation. IEEE Transactions on Broadcasting, 2018, 64 (4), pp.883-899. 10.1109/TBC.2018.2811623 . hal-01877334

HAL Id: hal-01877334

https://hal-univ-rennes1.archives-ouvertes.fr/hal-01877334

Submitted on 19 Sep 2018

HAL is a multi-disciplinary open access archive for the deposit and dissemination of scientific research documents, whether they are published or not. The documents may come from teaching and research institutions in France or abroad, or from public or private research centers.
L'archive ouverte pluridisciplinaire HAL, est destinée au dépôt et à la diffusion de documents scientifiques de niveau recherche, publiés ou non, émanant des établissements d'enseignement et de recherche français ou étrangers, des laboratoires publics ou privés. 


\title{
Quasi-Optimal Tone Reservation PAPR Reduction Algorithm for Next Generation Broadcasting Systems: A Performance/Complexity/Latency Tradeoff With Testbed Implementation
}

\author{
S. S. Krishna Chaitanya Bulusu , Member, IEEE, Matthieu Crussière, Member, IEEE, \\ Jean-François Hélard, Senior Member, IEEE, Ralph Mounzer, Student Member, IEEE, \\ Youssef Nasser, Senior Member, IEEE, Olivier Rousset, and Alain Untersee
}

\begin{abstract}
High peak-to-average power ratio (PAPR) of the transmitted signal is a serious issue in multicarrier communication systems, such as second generation terrestrial digital video broadcasting (DVB-T2) systems. These large fluctuations prevent feeding the high power amplifier at an operating point near its non-linear saturation region thereby lowering its power efficiency. In recent years, tone reservation (TR) PAPR reduction techniques have been deeply studied and included in the DVB-T2 and the American digital video broadcasting (ATSC 3.0) specifications. It is based on a gradient iterative approach where, at each iteration, a predefined kernel is used to reduce one peak in time domain. In this paper, a novel TR PAPR reduction technique namely individual carrier allocation for multiple peaks (ICMPs) that is based on a new kernel signal is proposed. This algorithm, compatible with the DVB-T2 standard, offers better performance than the gradient-based DVB-T2 algorithm but suffers with increased complexity for higher modes of DVB-T2 and ATSC 3.0 as the number of required iterations is equal to the number of reserved tones. To overcome this issue, we propose an improved ICMP technique, called grouped ICMP defined with this new kernel. The main principle of this new algorithm consists in dividing the reserved tones into $G$ groups. This highly reduces the number of iterations, now equal to the number of groups, and thereby the latency. An in-depth performance analysis has been done by implementing our algorithm on a testbed platform with real power amplifiers. Both the simulation and experimental results demonstrated that the proposed PAPR reduction algorithm offers very good performance/complexity/latency tradeoff.
\end{abstract}

This work was sup-ported in part by the European Eurostars Greentea Project under Grant 10433, and in part by PAPRICA Project. (Corresponding author: S. S. Krishna Chaitanya Bulusu.)

S. S. K. C. Bulusu was with the Department of Digital Communications, IETR-INSA, UMR CNRS 6164, 35000 Rennes, France. He is now with the Electrical and Electronics Engineering Department, Mahindra École Centrale, Hyderabad 500043, India (e-mail: sri-satish-krishna.bulusu@insa-rennes.fr).

M. Crussière, J.-F. Hélard, and R. Mounzer are with the Department of Digital Communications, IETR-INSA, UMR CNRS 6164, 35000 Rennes, France.

Y. Nasser is with the Department of Electrical and Computer Engineering, American University of Beirut, Beirut 1107 2020, Lebanon.

O. Rousset and A. Untersee are with the Research and Development Team, Teamcast, 35769 Saint-Grégoire, France.
Index Terms-OFDM, broadcasting, peak-to-average power ratio, power control, DVB-T2, ATSC 3.0, non-linear HPA, modulation error ratio, tone reservation, computational complexity, kernel phase optimization, latency.

\section{INTRODUCTION}

D URING the last three decades, multicarrier modulation has attracted a lot of attention among the scientific community in the field of telecommunications and terrestrial broadcasting. Orthogonal frequency division multiplexing (OFDM) technique, is a key technology which has extensively been deployed in wireless communication systems such as DVB, WiFi, WiMAX and LTE, primarily for its ability to cope with frequency selective channels. However, one main drawback of OFDM systems remains in its high peak-to-average power ratio (PAPR) related to the large fluctuations of the time domain signal and resulting from the large number of independently modulated subcarriers.

Indeed, due to the non-linearity of the input-output characteristics of the high power amplifier (HPA), the amplification in the non-linear (NL) region of multicarrier signals with high PAPR, leads to in-band and out-of-band distortions. The inband distortions degrade the link performance (such as bit error rate (BER)), while out-of-band distortions lead to interferences with adjacent channels and break the spectral mask. Consequently, the power level of the input multicarrier signal of the HPA needs to be reduced; a large number of dBs, roughly equivalent to the PAPR level of the input signal, must be backed-off. The main disadvantage of such power back-off is the decrease in the power efficiency of the HPA.

The objective of PAPR reduction is to decrease the fluctuations of the transmitted signal in order to exploit the HPA at an operating point closer to the saturation region where its power efficiency is maximized. Various approaches have been proposed and summarized in [1] and [2] to mitigate the PAPR of OFDM signals. Clipping and filtering [3], coding [4], partial transmit sequence [5] or selected mapping [6], are the most popular reduction techniques. The main drawback of these methods is their high complexity with limited gain and the 
necessity to transmit, for some of them, side information to the receiver.

In recent years, active constellation extension (ACE) [7] and tone reservation (TR) techniques [8] have been studied by many researchers because they offer the highest potential and do not need to transmit any side information. In particular, these two PAPR reduction techniques have been selected in various standards such as digital video broadcasting - second generation (DVB-T2) [9], DVB for next generation handheld (DVB-NGH) and more recently the American digital video broadcasting standard (ATSC 3.0) [10].

The TR concept introduced by Tellado [8] relies on the dedicated usage of a subset of subcarriers for PAPR reduction purposes. This subset of so-called reserved tones is used to generate a kernel signal added to the original one and resulting to a transmitted signal which has a lower PAPR than the original one. The computation of the complex values assigned to the reserved tones can be computed iteratively. For instance, the DVB-T2, DVB-NGH and ATSC 3.0 broadcasting specifications include a gradient-based iterative algorithm for TR PAPR reduction. At each iteration, such an algorithm detects the highest peak of the time domain signal, circularly shifts a copy of the original kernel in order to make kernel's peak coincide with that peak, scales the kernel amplitude and adjusts its phase in such a way that its sum with the original signal reduces the detected peak amplitude. The same process is repeated to detect and reduce the signal peaks, one by one. This peak-by-peak approach based algorithm predefines an amplitude threshold $V_{\text {clip }}$, and exits when all signal peaks fall below $V_{\text {clip }}$ or when the maximum number of iterations has been reached. This TR algorithm adopted by the DVB-T2 and DVB-NGH standards and described in [9] does not offer a sufficient performance/complexity trade-off to be implemented in present DVB-T2 modulators. That is why, to the best of our knowledge, the TR adoption in the world of the DVBT2 commercial modulator suppliers is less prevalent except a few [11]. This shows that this technology is not so mature from cost-effective implementation point of view.

These last years, multiple researches have focused on enhancing the performance of TR based algorithms. In [12] one TR solution called one kernel one peak (OKOP) was proposed consisting in changing the existing based kernel definition while remaining fully compatible with the DVB-T2 standard. An enhanced selection of the peaks and a dynamic computation of the threshold were developed to optimize the DVB-T2 TR algorithm. However, the OKOP kernels suffer from the same disadvantage as the DVB-T2 kernels, both being complex to generate in real-time (RT) or require considerable memory space.

Lately, based on least squares approximation, a TR method with fast convergence for PAPR reduction has been proposed in [13]. This method is capable of generating the optimal peak canceling signals with fast convergence and requires only two iterations to offer almost the same performance as that of the clipping control method. An interesting TR method is suggested in [14]. However, it requires an additional IFFT operation and a matrix inversion operation with a matrix size equal to the square of the number of reserved tones.
Rosati et al. [15] proposed, using the DVB-T2 reserved tones, a PAPR reduction method named as rotation invariant subcarrier mapping offering similar performance as that of DVB-T2 TR method. Recently, TR with a power constraint gaining currency, has been discussed in [16]. A TR method based on sphere encoding with power constraint has been proposed in [17]. However, these techniques either add complexity or offer reduced PAPR gain.

In this paper, we propose two novel PAPR TR reduction techniques based on a newly defined kernel signal which are implementable and compatible with the DVB-T2 standard. This proposed kernel is defined to deal with the reduction of multiple peaks at each iteration while optimizing the phase computation of each reserved tone. Two algorithms respectively named individual carrier allocation for multiple peaks (ICMP) and grouped individual carrier allocation for multiple peaks (GICMP) are described. Those algorithms lead to a very good usage of the available power of the reserved tones and then offer very good performance in terms of modulation error ratio (MER) and complementary cumulative density function (CCDF) of PAPR. The complexity analysis carried out in this paper shows that the GICMP algorithm offers a very good performance/complexity/latency trade-off. In addition, we provide measurements performed with a real testbed including a DVB-T2 generator equipped with the proposed GICMP PAPR reduction algorithm, a digital pre-distortion module and a NL HPA. The obtained results have confirmed the very high potential of our algorithm, which is fully compatible with existing DVB-T2, DVB-NGH and ATSC 3.0 standards.

The rest of the paper is organized as follows: Section II reminds the main PAPR minimization issues and the state of the art TR PAPR reduction algorithms. In Section III, the new ICMP and GICMP TR algorithms with the new kernel definition are described and compared to the state of the art algorithms. Section IV deals with the performance optimization of the proposed solutions, based on simulation results. Later, in Section $\mathrm{V}$, the measurements in terms of MER gain and energy consumption with a real testbed platform are presented. Finally, conclusions are drawn in Section VI.

\section{PAPR MINIMIZATION ISSUES}

\section{A. OFDM System Model}

Fig. 1 presents the structure of a typical OFDM transmitter. Let $\mathbf{X}=\left[X_{0}, \ldots, X_{n}, \ldots, X_{N-1}\right]$ be a sequence of complex symbols to be transmitted over the $N$ subcarriers of an OFDM system. Then, the continuous-time OFDM baseband signal $x(t)$ with sampling period $T$ can be written for each OFDM symbol of duration $T_{S}=N T$ as

$$
\begin{aligned}
x(t) & =\mathcal{F}\{\mathbf{X}\}, \\
& =\frac{1}{\sqrt{N}} \sum_{k=0}^{N-1} X_{k} e^{j 2 \pi \frac{k t}{N T}}, 0 \leq t<N T,
\end{aligned}
$$

where $\mathcal{F}\{$.$\} is the OFDM modulation function and j=\sqrt{-1}$. 


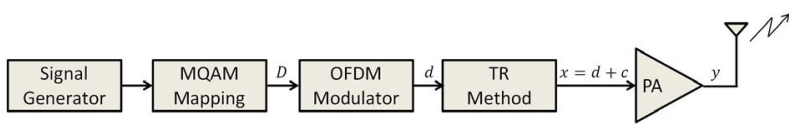

Fig. 1. A typical OFDM transmitter.

\section{B. HPA Model}

HPAs are crucial components in modern communication systems and are inherently NL. For amplifying $x(t)$, let us denote the continuous-time baseband equivalent of HPA output as $y(t)$. For an ideal HPA, the output signal $y(t)$ is nothing but an amplified input signal with a linear gain. However, in reality using a NL HPA, $x(t)$ will be amplified with a complex gain $\mathcal{A}\{$.\}. Thus $y(t)$ can be represented as

$$
y(t)=\mathcal{A}\{x(t)\} .
$$

In our analysis, we consider the well-known Rapp model which is commonly used for solid-state HPAs which do not apply any phase change to the input signal [18]. The amplification function of this model is defined as

$$
\mathcal{A}\{x(t)\}=\frac{x(t)}{\left(1+\left(\frac{|x(t)|}{v_{\text {sat }}}\right)^{2 p}\right)^{\frac{1}{2 p}}},
$$

where $p$ is the knee factor revealing the smoothness of transition from the linear region to the saturation region. Note that as the value of $p$ increases, the Rapp model approaches the so-called soft envelope limiter [19].

The high amplitude fluctuations of signals in OFDM systems pose a tough challenge to the RF design of HPAs. One solution to cope with NL distortions is to operate the HPA in its linear region. The operating point of a HPA is usually determined by the input back-off (IBO), often expressed in $\mathrm{dB}$, and defined as

$$
\mathrm{IBO}=10 . \log _{10}\left(\frac{v_{\text {sat }}^{2}}{\mathbb{E}\left[|x(t)|^{2}\right]}\right),
$$

where $v_{\text {sat }}$ is the saturation voltage of the HPA and $\mathbb{E}[$.$] is the$ expectation operator.

\section{PAPR Definition and Related Metrics}

The PAPR is a random variable which is a convenient parameter in measuring the sensitivity of a HPA non-linearity when a non-constant envelope input signal needs to be transmitted.

1) General PAPR Definition: The PAPR of a baseband signal is usually defined over an observation window of a given duration. In the case of OFDM signals, this duration is taken equal to the duration $T_{s}$ of OFDM symbols. The PAPR of the continuous-time base-band signal $x(t)$ transmitted during a symbol period $T$ is defined by

$$
\operatorname{PAPR}_{x(t)}=\frac{\|x(t)\|_{\infty}^{2}}{\frac{1}{T} \int_{0}^{T}\|x(t)\|_{2}^{2} \cdot d t},
$$

where $\|\cdot\|_{\infty}$ and $\|\cdot\|_{2}$ denote infinity and Euclidean norms, respectively.
2) $C C D F$ : The CCDF of PAPR is an useful parameter to analyze the PAPR and is most commonly used in the literature. The CCDF of PAPR of a signal quantifies how frequent the PAPR exceeds a given threshold value $\gamma$ (in $\mathrm{dB}$ ). It is defined as [20]

$$
C C D F_{x(t)}(\gamma)=\operatorname{Pr}\left(P A P R_{x(t)}>\gamma\right),
$$

where, $\operatorname{Pr}($.$) denotes probability function. It is well known that$ the probability that PAPR exceeds a given threshold increases when the number of subcarriers becomes larger in an OFDM system.

CCDF of PAPR is a very popular notion, but it has one severe drawback. It measures a certain clipping probability which ensures that at least one peak within the observation window has an important amplitude and is likely to undergo severe distortion, but gives no information on how many samples in that observation window are distorted. Thus, CCDF alone is not sufficient to predict the NL effects of HPA on signals as it do not take into account any additional peaks of lower amplitude. In practical scenarios, it is of greater interest to know how many samples have a certain level and are thus susceptible to be distorted. Indeed, severe clipping of one single peak in an observation window has negligible effect on the spectrum shape and on the quality of the transmitted signal, while even a mild distortion of large number of samples might have unacceptable consequences. From this point of view, it is important to consider a more refined analysis taking into account all the signal samples.

The MER metric defined in the sequel gives additional insights for performance evaluations.

3) MER: The MER is measured in baseband to quantify the performance of a digital radio transmitter or receiver in a communication system using digital modulation as it indicates the signal deterioration even before the BER result turns bad. The European Telecommunications Standards Institute (ETSI) defines the $\mathrm{MER}^{1}$ as one of the measurement guidelines for DVB systems [21] due to the fact that it reflects the quality of digital video signals directly. The MER computation is a figure of merit for system performance by comparing the actual location of a received symbol vector to its ideal location. It is defined in $\mathrm{dB}$ as

$$
\operatorname{MER}\{\mathbf{X}, \widehat{\mathbf{X}}\}=10 \log _{10}\left(\frac{\|\mathbf{X}\|_{2}^{2}}{\|\mathbf{X}-\widehat{\mathbf{X}}\|_{2}^{2}}\right) .
$$

where $\mathbf{X}$ is the ideal symbol vector measured at the input of the amplifier and $\widehat{\mathbf{X}}$ is measured at the output of the HPA. So, it is a ratio of the root mean square power of $\mathbf{X}$ to the power of the symbol error vectors $(\mathbf{X}-\widehat{\mathbf{X}})$. Thus, MER gives an indication of the ability of the receiver to correctly decode the signal.

The denominator in (7) indicates the energy of the distortion, which can also be related in time-domain to the amount of energy above a given threshold. It depends on the HPA response, hence affected by the saturation effects. Indeed, the MER reveals the distortion of the constellation points induced

\footnotetext{
${ }^{1}$ Note that MER and the error vector magnitude are closely related and one can generally be computed from the other.
} 
by the NL effects of the HPA and do not solely depend on the amplitude of the highest peak as CCDF of PAPR does. Hence, we propose in this paper to consider the MER instead of CCDF of PAPR, as the main parameter in quantifying the performance of PAPR reduction techniques. Nevertheless, the CCDF of PAPR is also given for the sake of clarification and comparison.

In addition, unlike CCDF of PAPR, MER is independent of the symbol length and then of the number of subcarriers. Regardless the fast Fourier transform (FFT) size, the percentage of samples being distorted for a given HPA, is always the same. This means that the energy of the time-domain OFDM signals above a given threshold is on average almost constant whatever the FFT size. This can be explained by the fact that the larger the FFT size, the higher the amplitude of the potential peaks in the time envelope of the signal, but at the same time the lower the probability of occurrence of these peaks. As a result, the measured MER does not depend on the FFT size.

To sum up, there is no direct relation between CCDF of PAPR and MER. The reduction of one large peak might highly reduce the PAPR while it has negligible effect on MER. On contrary, a slight reduction of multiple peaks in time-domain might have a slight change on PAPR while highly reduce the MER. Hence, these two figures of merit can be viewed as complementary. CCDF of PAPR is a measurement of the distribution of the signal before amplification, while MER measures the resulting distortion of the transmitted signal. Indeed, so, it is much more useful for the good dimensioning of the signal and for the choice of the operating point of the NL amplifier. That is why, MER is the most widely used figure of merit in the broadcasting industry.

\section{PAPR Minimization Using TR-Based Algorithms}

1) General Idea behind TR: The idea behind TR is to use a predefined subset of tones, referred to as peak reduction tones (PRTs), which add a signal in time domain to cancel large peaks. As shown in Fig. 2, the continuous-time OFDM baseband signal in time domain, after PRT insertion, becomes

$$
x(t)=c(t)+d(t)
$$

in which $c(t)$ is the peak cancellation signal obtained from the PRTs and $d(t)$ is the data signal. TR does not degrade the BER performance of the system, and thus can be categorized among downward compatible methods [22].

Let us consider that $R$ tones or PRTs are reserved for PAPR reduction of the OFDM system of $N$ subcarriers, where $R \ll N$. Calling $\mathcal{N}$ the set of locations of all tones in the multicarrier symbol, we define $\mathcal{B}$ as the PRT subset of these $R$ locations and $\mathbf{C}$ as the vector of $R$ peak reduction symbols transmitted on these positions and zeros elsewhere. Similarly, let the complement set $\mathcal{B}^{c}$ be the data tone (DT) subset of the useful data positions and $\mathbf{D}$ the vector of the $N-R$ associated transmitted data symbols and zeros elsewhere. So, we have $\mathcal{B} \cup \mathcal{B}^{c}=\mathcal{N}$ and $\mathcal{B} \cap \mathcal{B}^{c}=\emptyset$. The resulting signal to be transmitted can be represented in frequency domain as $\mathbf{X}$,

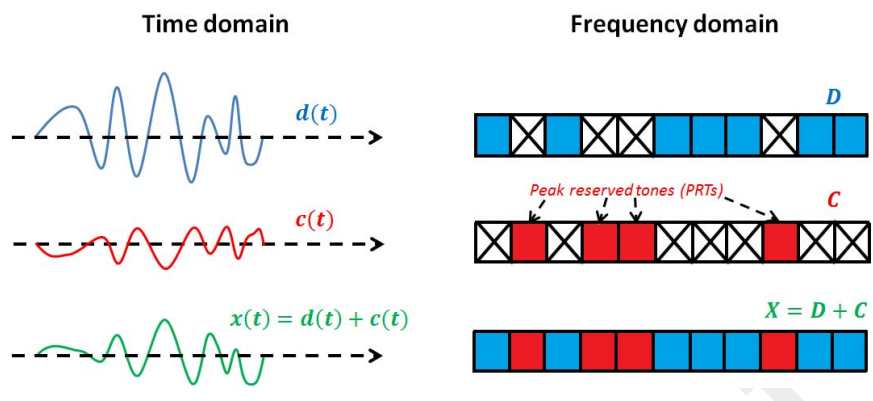

Fig. 2. Typical illustration of a TR scheme.

TABLE I Size of $R$ FOR DIFFERENT MODES IN DVB-T2 AND ATSC $3.0^{\dagger}$

\begin{tabular}{|c|c|c|c|c|c|c|}
\hline MODE & $1 \mathrm{~K}$ & $2 \mathrm{~K}$ & $4 \mathrm{~K}$ & $8 \mathrm{~K}$ & $16 \mathrm{~K}$ & $32 \mathrm{~K}$ \\
\hline$N$ & 1024 & 2048 & 4096 & 8192 & 16384 & 32768 \\
\hline$R$ & 9 & 18 & 36 & 72 & 144 & 288 \\
\hline
\end{tabular}

$\dagger$ ATSC 3.0 has only $8 \mathrm{~K}, 16 \mathrm{~K}$ and $32 \mathrm{~K}$ modes.

given by

$$
\mathbf{X}=\mathbf{C}+\mathbf{D}= \begin{cases}C_{k}, & k \in \mathcal{B}, \\ D_{k}, & k \in \mathcal{B}^{c},\end{cases}
$$

where $\mathbf{C}$ is 0 for $k \in \mathcal{B}^{c}, \mathbf{D}$ is 0 for $k \in \mathcal{B}$ and $0 \leq k<N$.

We denote $\kappa$ as the percentage of the subcarriers dedicated for PAPR reduction. For example, in DVB-T2 [9] and ATSC 3.0 [10] standards, $\kappa=1$, i.e., $1 \%$. The number of reserved tones for PAPR reduction (i.e., PRTs) for different OFDM symbol sizes is given in Table I.

Then, in a generic way the TR optimization problem can be stated as below

$$
\begin{array}{cl}
\min _{\mathbf{C}} & \tau \\
\text { subject to: } & \|d(t)+\mathcal{F}\{\mathbf{C}\}\|_{\infty}^{2} \leq \tau,
\end{array}
$$

where $\tau$ is the maximum peak power of $x(t)$.

Some of the solutions proposed in the literature to optimize $\mathbf{C}$ are briefly discussed in the following paragraphs.

2) $Q C Q P$ Solution: The TR optimization problem in (10) is a quadratically constrained quadratic program (QCQP) [23], which is a well studied convex problem. QCQP is an optimization problem in which both the objective function and the constraints are quadratic functions. The solution of the QCQP provides the optimal values to assign to the PRTs in order to obtain the lowest PAPR signal. Solving (10) requires very high computational complexity. Due to this reason, even though QCQP yields to the optimal result, we probe for other sub-optimal approaches that are feasible to implement. Many free software solvers exist to solve the convex optimization problem among which SeDuMi, YALMIP, CVX [24]. All these are very easy to use. CVX have been chosen in the simulations of this paper due to its ease of use and fast obtention of results.

3) Sub-Optimal Solutions: Sub-optimal approaches converge faster than the optimal QCQP solution. Among suboptimal approaches, the gradient search [23] solution is one of the most promising, which have been extensively studied for optimization purposes. Gradient-based solution is a 
TABLE II

ORDER OF COMPLEXITY FOR DIFFERENT SOLUTIONS

\begin{tabular}{|c|c|c|}
\hline Solution & QCQP & Gradient \\
\hline Complexity & $\mathcal{O}\left(R N^{2}\right)$ & $\mathcal{O}(N)$ \\
\hline
\end{tabular}

sub-optimal solution of the QCQP method in (10), which is proposed in DVB-T2 standard. It is based on the gradient iterative method that cancels out the signal peaks by a set of impulse-like kernels. The reference kernel signal denoted is a Dirac-like pulse defined by

$$
\begin{aligned}
\mathcal{K} & =\frac{N}{R} \mathcal{F}\left\{\mathbf{1}_{T R}\right\}, \\
\mathbf{1}_{T R} & = \begin{cases}1, & k \in \mathcal{B}, \\
0, & k \in \mathcal{B}^{c} .\end{cases}
\end{aligned}
$$

where $\mathbf{1}_{T R}$ is a column vector of order $N \times 1$. For optimal performance, the generated kernel should be designed to be as close as possible to a discrete-time impulse. At the start of this gradient method, the kernel signal $\mathcal{K}$ presents a peak at position 0 . For each iteration, the peak position $k$ of the initial signal is detected. Reference signal is shifted by $k$ positions in order to allow the reduction of peak signal to a predefined clipping value $V_{\text {clip }}$. The same process is then repeated to detect and reduce another signal peak. The algorithm exits when the maximum number of iterations $l$ has been reached or when there are no more signal peaks above a predefined threshold.

It has to be noted that this proposed solution in DVB-T2 does not specify the boost factor of each dedicated PRT but specifies the maximum permitted power of these PRTs.

4) Orders of Complexity of Different Solutions: The order of complexity of these two solutions has been summarized in Table II. Evidently, QCQP has very high computational complexity and yields to the optimal result. On the contrary, gradient method offering sub-optimal result has the least complexity, which is feasible for practical implementation. Interestingly, its complexity is independent of the size of $R$.

\section{E. PAPR Reduction and Power Control}

1) Impact of Power Control on PAPR Reduction: In practice, the existing DVB-T2 standard implementing PAPR reduction techniques specifies a maximum power $P_{\max }$ to be allocated to the PRTs in comparison with the power $P_{\text {data }}$ allocated to the DTs. The power control (PC) level denoted by $\lambda$, is equal to the difference in $\mathrm{dB}$ between the maximum PRT power and the mean DT power. It can be expressed mathematically as

$$
\max _{k \in \mathcal{B}}\left|C_{k}\right|^{2} \leq \Gamma \cdot P_{\text {data }},
$$

where $\Gamma=10^{\frac{\lambda}{10}}$ is the power level gap and $P_{\max }=\Gamma \cdot P_{\text {data }}$. $\lambda=10 \mathrm{~dB}$ is specified in DVB-T2 standard. An illustration of its impact in the frequency domain of the transmitted signal is shown in Fig. 3.

Due to the introduction of PC, an additional constraint gets added to (10). So, for $0 \leq k<N$, the modified TR optimization problem in the context of DVB-T2 can

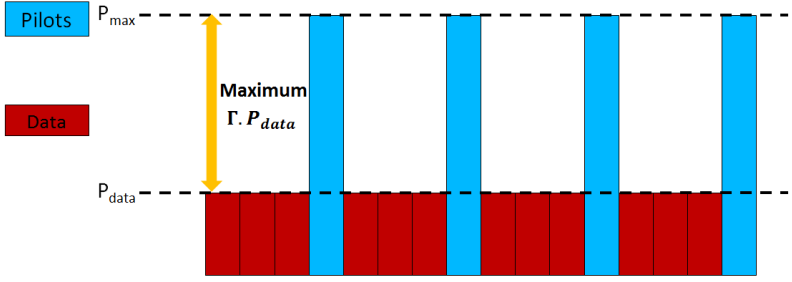

Fig. 3. Illustration of PC for TR in frequency domain. $\Gamma=10$ is imposed in DVB-T2 standard.

be written as

$$
\min _{\mathbf{C}} \tau
$$

subject to: $\|d(t)+\mathcal{F}\{\mathbf{C}\}\|_{\infty}^{2} \leq \tau$

$$
\left\{\left\|C_{k}\right\|_{\infty}^{2}\right\}_{k \in \mathcal{B}} \leq \Gamma\left\{\frac{\left\|D_{k}\right\|_{2}^{2}}{N-R}\right\}_{k \in \mathcal{B}^{c}},
$$

where

- $\left\{C_{k}\right\}_{k \in \mathcal{B}}$ are the elements of PRT subset of size $R$,

- $\left\{D_{k}\right\}_{k \in \mathcal{B}^{c}}$ are the elements of DT subset of size $N-R$.

Actually, new optimization problem in (14) with PC is also a QCQP. Indeed, the RHS of the second constraint in (14) is a constant. The formulation proposed in LHS of the second constraint, $\left\{\left\|C_{k}\right\|_{\infty}^{2}\right\}_{k \in \mathcal{B}}$ is a L-infinity norm minimization. Thus, the second constraint is a quadratic function and (14) is QCQP.

a) Impact on receiver sensitivity loss: Higher $\lambda$ means that more power has to be allocated for PRTs. At constant total transmitted output power $P_{\text {out }}$, this increase in PRT power for PAPR reduction implies that less power is assigned to the data subcarriers, thereby inducing some receiver sensitivity loss, denoted as $\eta$. It can be calculated in $\mathrm{dB}$ for different $\lambda$ values as

$$
\eta=10 \log _{10} \frac{(100-\kappa)+\kappa \times \Gamma}{100} .
$$

We recall, $\kappa$ is the percentage of the subcarriers dedicated to PAPR reduction (i.e., $\kappa=1$ in the case of DVB-T2). Thus, by lowering $\lambda$, we can reduce $\eta$. For $\lambda=\{3,5,10\} \mathrm{dB}, \eta$ has been summarized in Table III.

On the contrary, when $P_{\text {data }}$ is kept unchanged, then higher $\lambda$ value means that more power has to be allocated to the PRTs, thereby increasing the total transmitted output power $P_{\text {out }}$ by a factor equal to $\eta$.

b) Impact on CCDF of PAPR and MER: The impact of PC has been assessed through simulations for the QCQP algorithm using 64 QAM in the $2 \mathrm{~K}$ mode of the DVB-T2 standard. The impact of $\lambda$ on the CCDF and MER are shown in Fig. 4 and Fig. 5. The CCDF of PAPR at $10^{-3}$ value have been summarized in Table III. For $\lambda=\{3,5,10\} d B$, there is relatively increasing degradation of PAPR reduction by $\{3.27,2.58,1.39\} \mathrm{dB}$ respectively at $10^{-3}$ value of CCDF of PAPR w.r.t. QCQP without any PC constraint (i.e., $\lambda=\infty \mathrm{dB}$ ).

The MER results are given versus the used IBO of the HPA, which is modeled as a Rapp model with a knee factor, $p=10$. The IBO gain can be deducted by comparing the MER values of the signal with and without PAPR reduction. A target value of MER above $34 \mathrm{~dB}$ is considered in simulation. The reason 


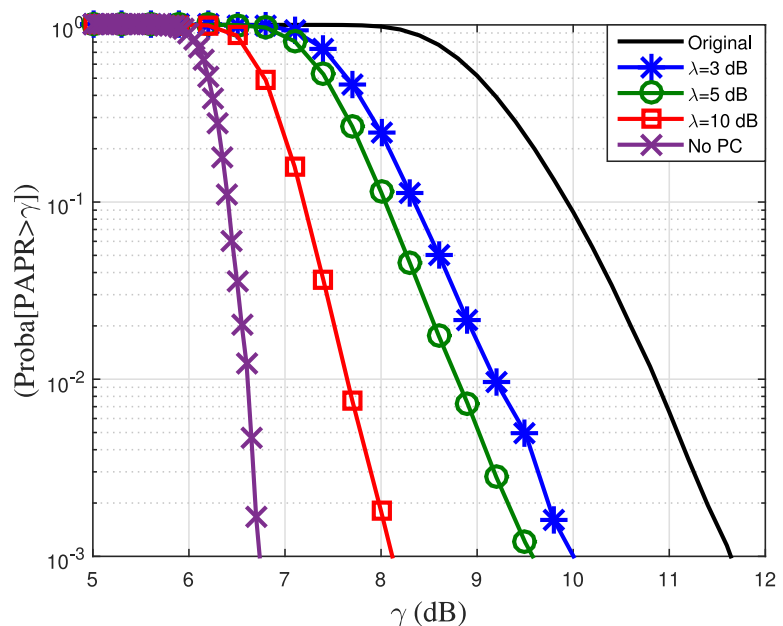

Fig. 4. CCDF of PAPR with QCQP for DVB-T2 with 64 QAM in $2 \mathrm{~K}$ mode.

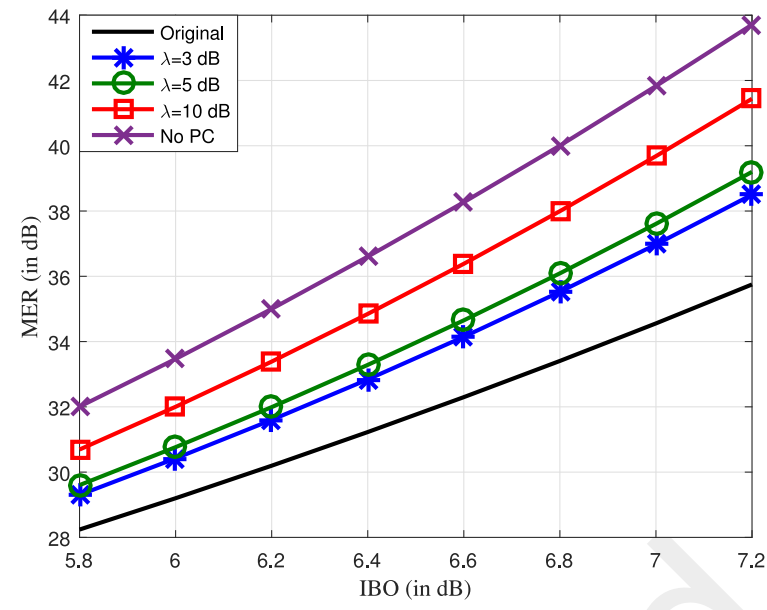

Fig. 5. MER with QCQP for DVB-T2 with 64 QAM in 2K mode with Rapp model HPA, $p=10$.

TABLE III

CCDF OF PAPR AT $10^{-3}$ VALUE FOR QCQP AND RECEIVER SENSITIVITY LOSS $\eta$ FOR DIFFERENT $\lambda$, IN $2 \mathrm{~K}$ MODE FOR $\kappa=1$

\begin{tabular}{|c|c|c|}
\hline$\lambda$ & CCDF OF PAPR AT $10^{-3}$ & $\eta$ \\
\hline 3 & $10.0 \mathrm{~dB}$ & $0.043 \mathrm{~dB}$ \\
\hline 5 & $9.58 \mathrm{~dB}$ & $0.093 \mathrm{~dB}$ \\
\hline 10 & $8.12 \mathrm{~dB}$ & $0.374 \mathrm{~dB}$ \\
\hline$\infty$ & $6.73 \mathrm{~dB}$ & - \\
\hline
\end{tabular}

for this choice is that in DVB-T transmission, a MER less than $32 \mathrm{~dB}$ is deemed to be a possible transmission failure [25] and the same can be inferred in the case of DVB-T2. Generally, a guard of $2 \mathrm{~dB}$ is usually taken in practice. Moreover, a MER above $34 \mathrm{~dB}$ assures a transmitter RF coverage almost similar to that of the theoretically achievable RF coverage limit [26]. Similarly, MER gain has been deducted in a similar manner with a target value of $6.4 \mathrm{~dB}$ IBO. The MER for different $\lambda$ values have been summarized in Table IV.

Both the CCDF of PAPR and MER highlight the impact of PC constraint in the design of efficient PAPR reduction algorithms. From these results, it is concluded that with this optimal QCQP algorithm the impact of $\lambda$ is far from being negligible. We can notice that optimal solution is achieved
TABLE IV

RElative Gains FOR Signal With QCQP W.R.T. THE ORIGINAL ONE FOR RAPP MODEL OF HPA, $p=10$, IN $2 \mathrm{~K}$ MODE

\begin{tabular}{|c|c|c|}
\hline$\lambda$ & $\begin{array}{c}\text { IBO gain w.r.t. } \\
\text { original signal }^{\dagger}\end{array}$ & $\begin{array}{c}\text { MER gain w.r.t. } \\
\text { original signal }^{\star}\end{array}$ \\
\hline 3 & $0.345 \mathrm{~dB}$ & $1.72 \mathrm{~dB}$ \\
\hline 5 & $0.419 \mathrm{~dB}$ & $2.19 \mathrm{~dB}$ \\
\hline 10 & $0.619 \mathrm{~dB}$ & $3.62 \mathrm{~dB}$ \\
\hline$\infty$ & $0.843 \mathrm{~dB}$ & $5.37 \mathrm{~dB}$ \\
\hline
\end{tabular}

$\dagger$ : The original signal has IBO of $6.903 \mathrm{~dB}$ at $34 \mathrm{~dB}$ of MER

$\star$ : The original signal has MER of $31.23 \mathrm{~dB}$ at $6.4 \mathrm{~dB}$ of IBO

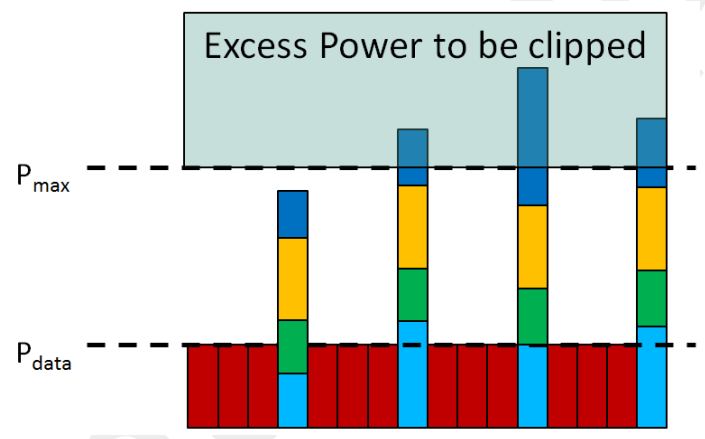

Fig. 6. An illustration of FCPC scheme in frequency domain

when PC constraint is absent and PC leads to some degradation of PAPR reduction gain. The PAPR is inversely proportional to $\lambda$.

In that perspective, two different techniques to control the power allocated by the TR algorithm are discussed in the next two following paragraphs. In this paper, we do not consider all the algorithms mentioned in the state-of-art, because most of them either offer very good performance, albeit with very high complexity or offer sub-optimal performance similar to that of DVB-T2 TR algorithm. So, we take into account only QCQP achieving optimal performance with very high complexity and the DVB-T2 TR algorithm offering sub-optimal performance with low complexity, in order to compare and propose new solutions that offer very good performance/complexity tradeoff.

2) Power Control Through Frequency-Domain Clipping: Early releases of the DVB-T2 standard [9] did not specify a PC scheme implementation. A straight-forward approach was to allow the TR algorithm to complete (i.e., execute all iterations) for a given OFDM symbol and then clip the amplitude of the subcarriers not meeting the power constraint. In this case, the $\mathrm{PC}$ is done on a symbol-by-symbol basis. This PC scheme is referred as frequency-domain clipping PC (FCPC) and is illustrated in Fig. 6. In the same figure, the power buildup at each iteration is shown with a different color. However, the PAPR reduction process is executed in time-domain, while the PC constraint is defined for each subcarrier in frequencydomain. This implies that the FCPC requires an additional inverse FFT (IFFT) to be computed.

3) Power Control Through Time-Domain Tracking: The processing of an OFDM symbol, including PAPR reduction, must be completed before the next symbol is received. This 


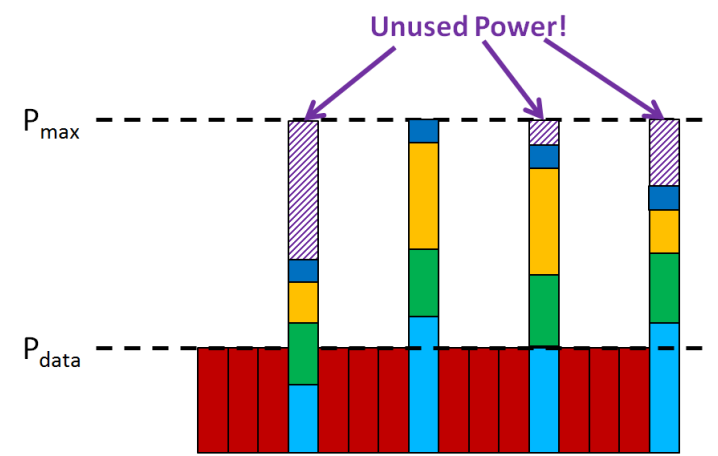

Fig. 7. An illustration of DVB-T2 TR algorithm in frequency domain.

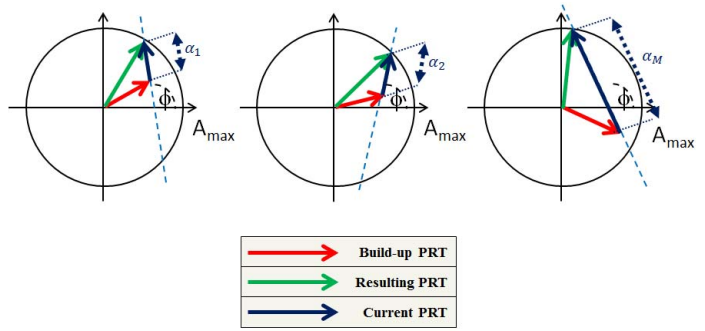

Fig. 8. PRT building up for each iteration in DVB-T2 TR algorithm.

makes it very challenging to fit in a second IFFT operation for PC purpose in addition to that already required for the OFDM symbol generation. To avoid this, the last version of the DVB-T2 standard [27] specifies a PC scheme that keeps track, iteration by iteration, of the power aggregated by each PRT and allocates power in order not to exceed the threshold. This PC scheme is referred as time-domain tracking PC (TTPC) or DVB-T2 TR algorithm and is illustrated in Fig. 7. It defines an amplitude scaling factor $\alpha_{k}$ that is applied to the kernel $\mathcal{K}$ at the $i^{\text {th }}$ iteration such that the $k^{\text {th }}$ PRT $C_{k}$, for $k \in \mathcal{B}$ exceeding the power limit is computed as follows

$$
\alpha_{k}^{(i)}=\sqrt{\left(A_{\max }\right)^{2}-\operatorname{Im}\left\{u_{k}^{*} r_{k}^{(i-1)}\right\}^{2}}+\operatorname{Re}\left\{u_{k}^{*} r_{k}^{(i-1)}\right\},
$$

where,

- $u_{k}=\frac{1}{c[0]} e^{-j 2 \pi\left(\left(\mathcal{B}(k)-p_{k}\right) \bmod N\right)} \cdot e^{-j \phi}$

- $c[0]$ is the peak of kernel $\mathcal{K}$ at position 0 ,

- $p_{k}$ is the position of the peak being processed,

- $\phi$ is the phase of the peak being processed,

- $A_{\max }$ is square root of the maximum power per PRT,

- $r_{k}^{(i-1)}$ is the value of $C_{k}$ after the previous iteration.

The geometric explanation of the above equations is shown in Fig. 8. The DVB-T2 TR algorithm aims at finding $\alpha_{k}$, in such a way that the resulting amplitude of the sum of the build-up PRT and current PRT is equal to $A_{\max }=\sqrt{P_{\max }}$. $\alpha_{k}$ is computed for every PRT. Without violating the PC, the amount of scaling that can be performed is given by

$$
\alpha=\min _{k \in \mathbf{B}}\left(\alpha_{k}^{(i)}\right)
$$

Fig. 8 shows the power build up after each iteration. The DVBT2 TR algorithm exits when at least one of the PRTs reaches the allowed power limit as shown in Fig. 7.
4) Impact on Performance and Complexity: FCPC outperforms DVB-T2 TR algorithm, both in MER and CCDF metrics. However, it requires an additional IFFT to be computed for each OFDM symbol, which is costly in terms of resource usage and causes additional processing delays. One main drawback of the DVB-T2 TR algorithm is that only a meager part of the available power of the reserved subcarriers is used, which explains the poor performance of this solution. Hence, there are possibilities to better exploit the allowed power while keeping simple implementation and reduced complexity. In the sequel, we will propose new kernel aiming at realizing both objectives.

\section{PAPR REDUCTION ALgORITHMS USING A NeW KeRnel DeFinition}

The DVB-T2 TR algorithm solution leaves unused a part of the power allocated to the PAPR reduction. The techniques presented in this section are based on a new kernel definition and new power to the PRTs in order to benefit from all the amount of power available for PAPR reduction. Hence, we introduce a first algorithm referred to as ICMP offering a good performance/complexity trade-off. Then, a second algorithm named GICMP is proposed in order to lower the latency of the previous one. Unlike the previous two algorithms which follow a peak-by-peak approach, these new schemes deal with multiple peaks at each iteration.

\section{A. New Kernel Definition Based on Individual Reserved Carriers}

Instead of a Dirac-type kernel as suggested by the DVBT2 specifications, it is possible to generate a comb-like one, associated to each PRT. By phase-shifting the kernel, we try to reduce the peaks of the data signal. This kernel is defined for every iteration $i$, as below

$$
C^{(i)}[k]= \begin{cases}A_{\max } e^{-j \phi}, & k \in \mathcal{B} \\ 0, & \text { else }\end{cases}
$$

where $\phi$ is the kernel phase. In time domain, the new kernel $c^{(i)}(t)$ is easily computed as follows

$$
c^{(i)}(n t / N)=\frac{A_{\max }}{N} e^{-j \phi} e^{-j \frac{2 \pi \mathcal{B}(i) n}{N}} \text {, for } 0 \leq n<N,
$$

where $\mathcal{B}(i)$ is $i^{\text {th }}$ element in the PRT subset $\mathcal{B}$ and thereby, $A_{\text {max }}$ is directly taken as the amplitude of the PRTs as in (18) in order to satisfy the PC constraint. Interesting characteristic of such a kernel is that it has a constant amplitude and the phase-shift between two consecutive samples is constant and is equal to

$$
\frac{c^{(i)}(n t / N)}{c^{(i)}((n-1) t / N)}=e^{j \frac{2 \pi \mathcal{B}(i)}{N}} .
$$

Owing to this latter property, the real-time generation of the new kernel requires a simple phase shift operation only, whereas a real-time kernel generation is required using FCPC and DVB-T2 TR algorithms. Furthermore, in the former an additional IFFT needs to be performed for the PC management which is not the case in the proposed approach due to the implicit PC through $A_{\max }$. 


\section{B. Kernel Phase Computation Optimization}

The FCPC and DVB-T2 TR algorithm take into account the phase of the highest detected peak in order to adjust the kernel phase $\phi$ in (16). The novelty in our approach is that it involves the identification of the optimal phase for kernel, which takes into account several peak positions. The computation of this optimal phase is discussed below.

1) Optimization Condition: The optimal phase is identified such that $S$ multiple peaks are reduced in a single iteration. Mathematically, the ICMP optimization problem can be stated as

$$
\min _{\phi, S} \sum_{S \in \mathcal{H}}\left\|d_{S}+c_{S} \cdot e^{-j \phi}\right\|_{\infty}^{2}
$$

The problem stated in (21) is a L-infinity norm minimization that aims at reducing the highest peak at each iteration. Solving (21) is not so easy as we need to optimize both $S$ and $\phi$, as the optimal solution to such L-infinity norm minimization problem cannot be solved analytically.

Therefore, we propose to decompose the problem in (21) into two sub-problems. While the first sub-problem is related to the optimal computation of $\phi$, the second one aims at finding an optimal selection of $S$. Therefore, we pose (21) alternatively, with an aim to reduce energy above a particular threshold, which is in line with the idea of maximizing the MER of the transmitted signal as discussed in section II.C.

The first sub-problem can be solved by fixing $S$ and then finding the optimal phase $\phi$ that minimizes the sum of the squares of these peaks above threshold. This can be mathematically formulated as a L-2 norm minimization for a given $S$ as

$$
\begin{aligned}
\min _{\phi} & \sum_{S \in \mathcal{H}}\left\|d_{S}+c_{S} \cdot e^{-j \phi}\right\|_{2}^{2}, \\
= & \min _{\phi} F(\phi),
\end{aligned}
$$

where $\mathcal{H}$ is the set of the $S$ highest peak positions of data signal $d(t)$. Then, $d_{S}$ and $c_{S}$ represent the finite subset of time samples corresponding to these positions for the data signal and the adding kernel (i.e., $d_{S} \subset d(t)$ and $c_{S} \subset c(t)$ ). We remind that problem stated in (22), instead of reducing the highest peak at each iteration, aims at reducing the energy above a particular threshold. By varying $S$, this threshold level is indirectly adjusted. The solution of the second sub-problem, i.e., the optimal choice of parameter $S$, will be obtained through simulation analysis in Section IV.

2) Optimal Phase Calculation: To solve (22), first, $S$ highest peaks are identified. Then, the optimal phase $\phi$ that minimizes the sum of the squares of these peaks, has to be computed by differentiating $F(\phi)$ and solving $\frac{\partial F}{\partial \phi}=0$. We found that $F(\phi)$ has minimum at

$$
\begin{aligned}
\phi & =\frac{3 \pi}{2}-\operatorname{atan} 2(\mathrm{~B}, \mathrm{~A}), \\
\text { where } A & =\sum_{s \in \mathcal{H}} \mathcal{R} e\left\{d_{S}\right\} \cdot \operatorname{R} e\left\{c_{S}\right\}+\mathcal{I} m\left\{d_{S}\right\} \cdot \operatorname{Im}\left\{c_{S}\right\}, \\
B & =\sum_{s \in \mathcal{H}} \mathcal{R} e\left\{d_{S}\right\} \cdot \operatorname{Im}\left\{c_{S}\right\}+\mathcal{I} m\left\{d_{S}\right\} \cdot \operatorname{Re}\left\{c_{S}\right\} .
\end{aligned}
$$

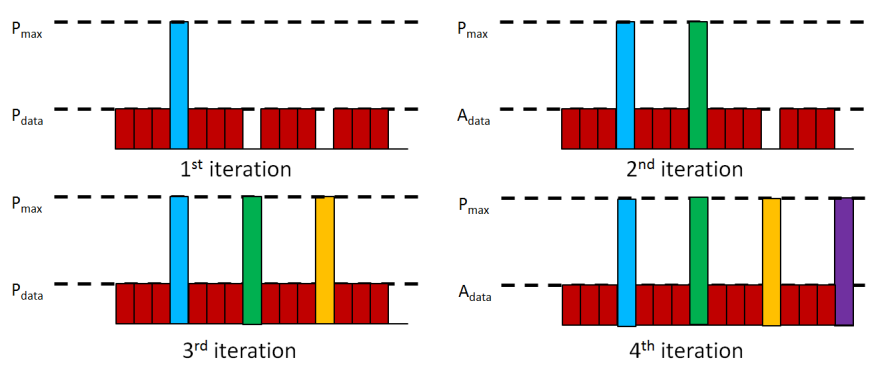

Fig. 9. Iterative power allocation of the PRTs with ICMP in the frequency domain.

From the definition of $A$ and $B$, it is evident that the computation of $\phi$ requires pre-computation and storage of only the $S$ samples of $c(t)$.

\section{The New ICMP Algorithm}

As anticipated by the new kernel definition, the main idea behind the ICMP technique is to target not just a single peak but multiple peaks in one iteration. In ICMP, the maximum number of iterations $(l)$ equals the number of available PRTs $(R)$. The relation between the new kernel definition and the iteration count in ICMP is illustrated in Fig. 9. In that figure we can notice that in frequency-domain, the kernel amplitude is set to the power constraint $P_{\max }$ as per (18). It means no explicit power control is required at each iteration in this approach since the power constraint is respected by design. The ICMP approach to solve (22) is summarized in Algorithm 1. The ICMP algorithm involves few important steps. First, for each PRT $S$ peaks are identified. Second, the kernel is computed in frequency domain as per (18) and its time-domain is obtained as per (19). Then, the optimal phase calculation is done as per (24). It is worth noting that ICMP has implicit FCPC as it ensures PC in frequency-domain. Thus, it is expected to better exploit the available power of the PRTs and then outperform DVB-T2 TR algorithm.

In ICMP, the final peak cancellation signal can be written as

$$
c(t)=\sum_{k=1}^{R} c^{(k)}(k t / N) e^{j \phi_{k}} .
$$

With ICMP, each iteration performs search of multiple peaks. Every peaks search traverses the whole signal, which means that the duration of each iteration is related to the length of one OFDM symbol. This peak search operation can be performed at a higher clock rate in order to minimize the induced delay. This induced processing delay mainly due to the peak search operation at each iteration, is termed as latency, which is then directly proportional to the number of iterations. Consequently, for the lower modes of DVB-T2 such as $2 \mathrm{~K}$, $4 \mathrm{~K}$ and $8 \mathrm{~K}$, the number of iterations remains acceptable (see Table I) resulting in a reasonable latency.

\section{The New GICMP Algorithm}

For higher modes such as $16 \mathrm{~K}$ and $32 \mathrm{~K}$ where 144 and 288 tones are reserved, carrying out ICMP algorithm becomes 


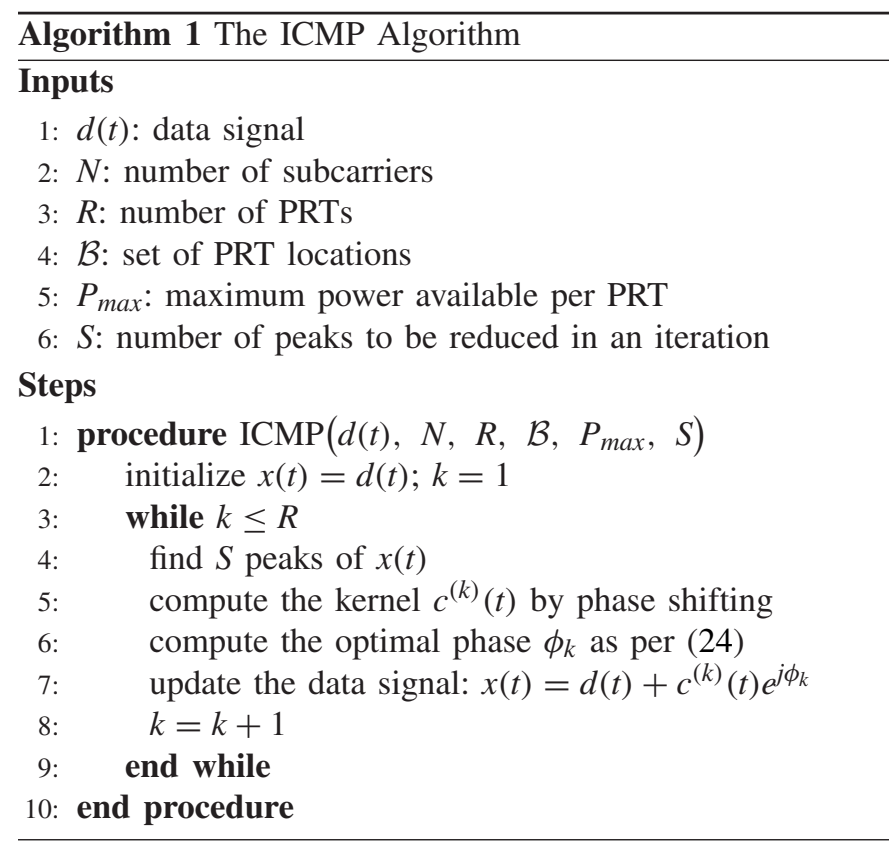

increasingly challenging. The resulting latency according to the number of PRTs may exceed the duration of one OFDM symbol depending on the used over-clock factor. To address this latency issue of the ICMP algorithm, we propose a new algorithm, namely GICMP, based on a grouping strategy of the PRTs. More precisely, the PRTs are grouped into $G$ groups as follows

$$
\begin{aligned}
\mathcal{B} & =\left\{\mathcal{B}_{1}, \ldots, \mathcal{B}_{G}\right\}, \\
\mathcal{B}_{i} & =\left\{C_{1+\frac{(i-1) R}{G}}, \ldots, C_{\frac{i R}{G}}\right\}, \text { for } 1 \leq i \leq G .
\end{aligned}
$$

Then, one peak search is executed per group of PRTs with GICMP, instead of one per PRT with ICMP. For instance, the iterative power buildup for an OFDM configuration with 32 subcarriers, 8 PRTs and $G=4$ groups is illustrated in Fig. 10. Note that, as with the ICMP algorithm the allocated power with GICMP is equal for each PRT to the maximum available level $P_{\max }$. The phase value applied to each PRT is computed as with the ICMP algorithm according to Eq. (24). The main interest of carrying out a peak search per group is to lower the latency which then becomes proportional to the number of groups.

The GICMP approach to solve (22) is summarized in Algorithm 2. First, $S$ peaks are searched for each PRT group. Then, following the same principle as with ICMP, the kernel and phase computations are carried out using (19) and (24) for each PRT of each group. Although the computation of the phases is based on a parallel processing, the phases of the PRTs of a same group are independent from each other and the number of phase value computations remains the same as with ICMP. The counterpart of the parallel processing is the increase of the computation complexity of each iteration. Finally, a trade-off between latency and computational complexity has to be studied as addressed in the simulation analysis of Section IV.
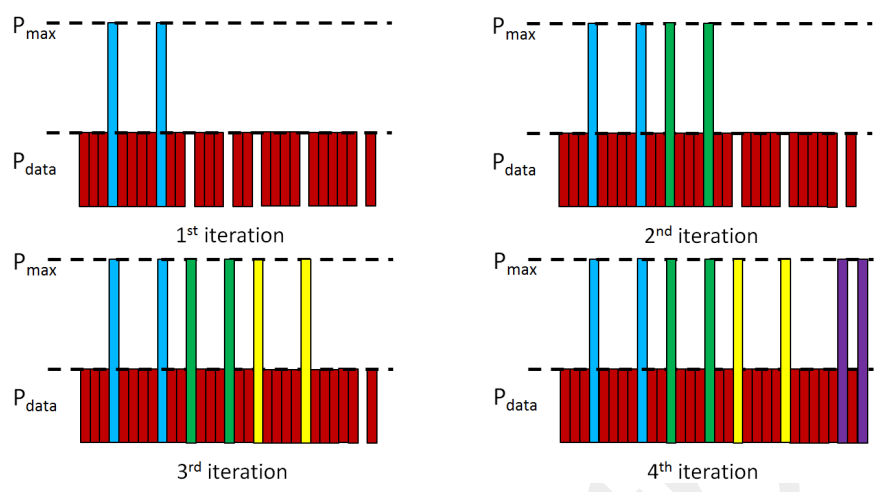

Fig. 10. Iterative power allocation of the PRTs with GICMP in the frequency domain.

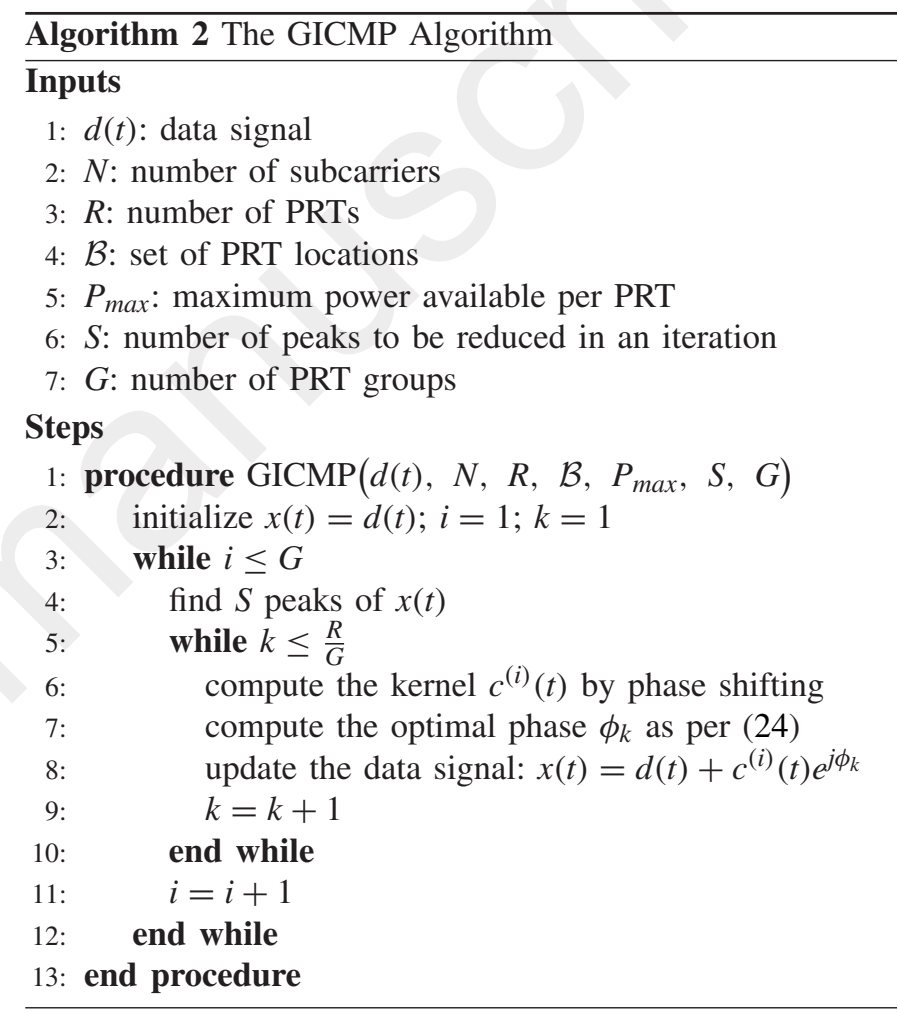

Thus, with GICMP, the final peak cancellation signal can be written as

$$
c(t)=\sum_{i=1}^{G} \sum_{k=1}^{\frac{R}{G}} c^{(i)}(k t / N) e^{j \phi_{k}} .
$$

\section{Algorithm Optimization Through SIMULATION ANALYSIS}

This section deals with performance of the different algorithms in order to optimize the choice of the parameters. More precisely, the objective is to determine the best performance/complexity/latency trade-off of the algorithms in the perspective of the testbed implementation.

To model the global response of the HPA preceded by a pre-distortion module in the final testbed, a Rapp model with a quite high value of the knee factor $p$ should be used for the simulations. This implicitly corresponds to a HPA with 


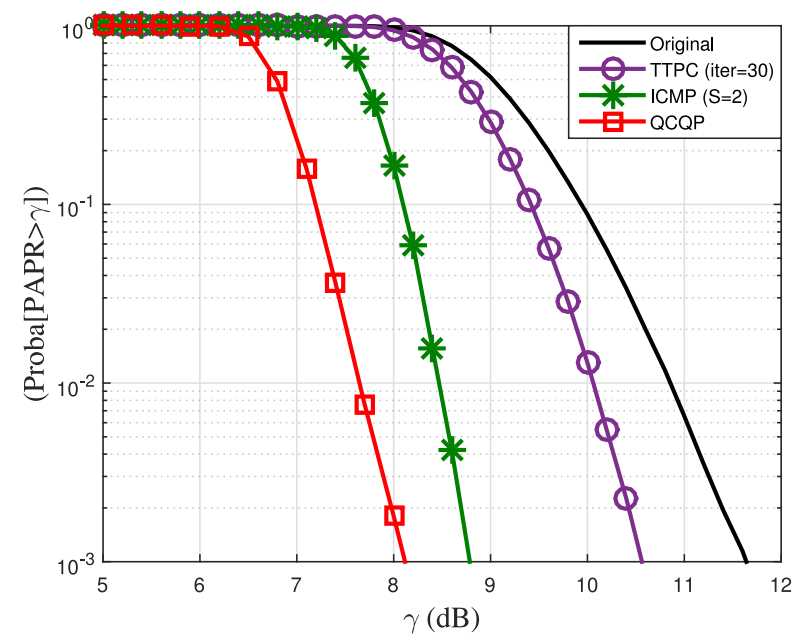

Fig. 11. CCDF of PAPR with QCQP, ICMP and TTPC for DVB-T2 with $64 \mathrm{QAM}$ in $2 \mathrm{~K}$ mode.

a larger linear range compared to a non-precorrected HPA. After various comparative tests between the testbed platform and the simulation chain, it turned out that a value of $p=10$ was a satisfactory choice. Also, 64 QAM constellations have been used for all modes of the DVB-T2 system.

\section{A. ICMP Algorithm Optimization}

1) Performance Comparison With State of the Art Algorithms in $2 \mathrm{~K}$ and $8 \mathrm{~K}$ Modes: As already mentioned, QCQP yields optimal performance at the price of huge computational complexity. The objective of these first results is to compare the performance of implementable solutions as the TTPC DVB-T2 algorithm and the new ICMP algorithm, to this optimal QCQP solution. For this comparison, $\lambda=10 \mathrm{~dB}$ has been selected and due to the prohibitive computational cost of QCQP, only $2 \mathrm{~K}$ and $8 \mathrm{~K}$ modes have been chosen.

In $2 \mathrm{~K}$ mode, the performances of QCQP, TTPC and ICMP with $S=2$ are presented in Fig. 11. In the case of TTPC (i.e., DVB-T2 TR algorithm), a clipping value $V_{\text {clip }}=7.2 \mathrm{~dB}$ is used with a maximum number of iterations $l=30$. The performance of QCQP serves as the lower bound of CCDF of PAPR for not only ICMP but also for any TR scheme with PC constraint. At $10^{-3}$ value of CCDF of PAPR, ICMP with $S=2$ not only outperforms TTPC of DVB-T2 but also has a low effective loss of PAPR w.r.t. QCQP equal to $0.68 \mathrm{~dB}$. In $8 \mathrm{~K}$ mode, the performances of CCDF of PAPR of QCQP, TTPC and ICMP with $S=4$ are presented in Fig. 12. As expected, ICMP offers better performance than the DVB-T2 TR algorithm.

2) ICMP Parameter Optimization: Fig. 13 shows the MER with the original signal along with QCQP and ICMP PAPR reduction for different DVB-T2 modes with various FFT sizes. $\lambda=10 \mathrm{~dB}$ and the Rapp model with $p=10$ are considered. For ICMP, the values of $S$ considered in $2 \mathrm{~K}, 4 \mathrm{~K}, 8 \mathrm{~K}, 16 \mathrm{~K}$ and $32 \mathrm{~K}$ modes are $\{8,32,64,120,200\}$, respectively. Owing to the huge complexity of QCQP, only $2 \mathrm{~K}, 4 \mathrm{~K}$ and $8 \mathrm{~K}$ modes are simulated. It can be noticed that original MER does not change with FFT size while the change is very meager in the

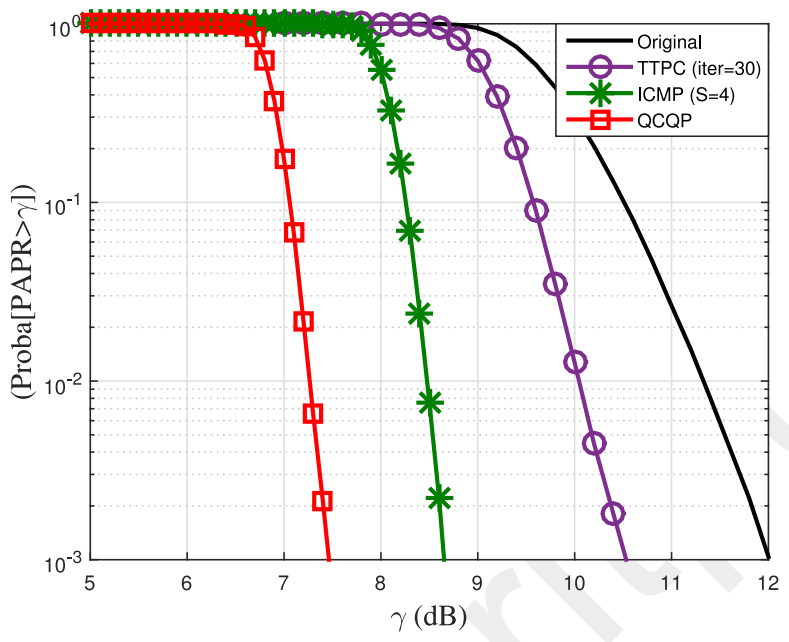

Fig. 12. CCDF of PAPR with QCQP, ICMP and TTPC for DVB-T2 with 64 QAM in $8 \mathrm{~K}$ mode.

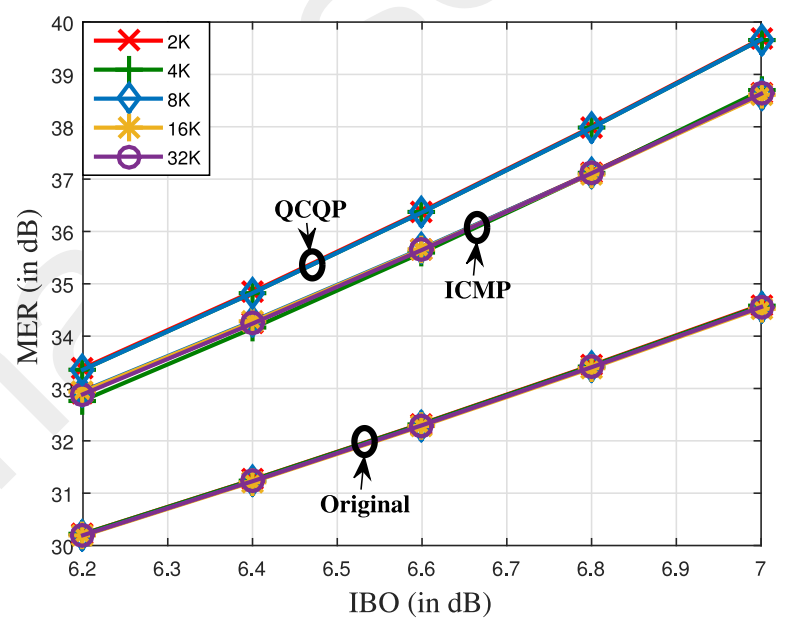

Fig. 13. MER for original signal along with QCQP and ICMP for DVB-T2 with 64 QAM for various FFT sizes with Rapp model HPA, $p=10$.

TABLE V

RELATIVE GAINS FOR ICMP W.R.T. THE ORIGINAL SIGNAL AND LOSS W.R.T. QCQP FOR RAPP MODEL HPA, $p=10$, IN 32K MOdE

\begin{tabular}{|c|c|c|c|}
\hline$S$ & $\begin{array}{c}\text { IBO gain w.r.t. } \\
\text { original signal }^{\dagger}\end{array}$ & $\begin{array}{c}\text { MER gain w.r.t. } \\
\text { original signal }^{\star}\end{array}$ & $\begin{array}{c}\text { MER loss w.r.t. } \\
\text { signal with QCQP }\end{array}$ \\
\hline 50 & $0.422 \mathrm{~dB}$ & $2.25 \mathrm{~dB}$ & $1.40 \mathrm{~dB}$ \\
\hline 100 & $0.494 \mathrm{~dB}$ & $2.70 \mathrm{~dB}$ & $0.92 \mathrm{~dB}$ \\
\hline 200 & $0.538 \mathrm{~dB}$ & $3.01 \mathrm{~dB}$ & $0.61 \mathrm{~dB}$ \\
\hline
\end{tabular}

$\dagger$ : The original signal has IBO of $6.903 \mathrm{~dB}$ at $34 \mathrm{~dB}$ of MER

$\star$ : The original signal has MER of $31.23 \mathrm{~dB}$ at $6.4 \mathrm{~dB}$ of IBO

$\ddagger$ : The signal with QCQP has MER of $34.85 \mathrm{~dB}$ at $6.4 \mathrm{~dB}$ of IBO

case of QCQP and ICMP. Thus, it can be inferred that when a TR algorithm reaches its optimal performance pertaining to its constraints, then its MER variation with FFT size becomes negligible. Consequently, for the following parts, QCQP in $8 \mathrm{~K}$ mode will be the reference mode, as the performance of QCQP in $8 \mathrm{~K}, 16 \mathrm{~K}$ and $32 \mathrm{~K}$ modes are the same.

In $32 \mathrm{~K}$ mode, ICMP with $S=\{50,100,200\}$ has been considered and its MER plot is shown in Fig. 14 in comparison with the DVB-T2 algorithm using 30 iterations. As observed in $32 \mathrm{~K}$ mode, increasing $S$ improves the MER for a given 


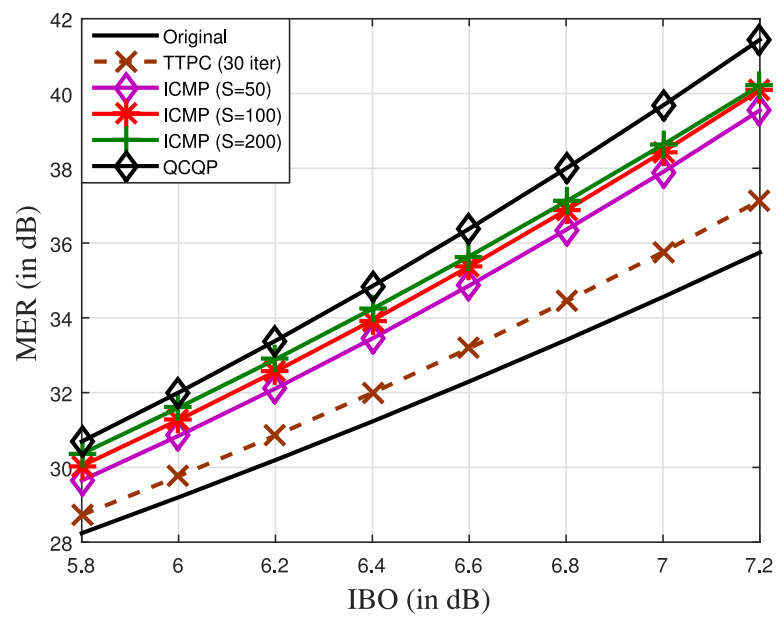

Fig. 14. MER with ICMP for DVB-T2 with 64 QAM in 32K mode with Rapp model HPA, $p=10$.

IBO. It is however noticed that, most of the potential gain is reached for $S=100$, any further increase in the value of $S$ yielding to a more moderate improvement. Hence, for $32 \mathrm{~K}$ mode, we suggest $S=100$ to be a wise choice in terms of performance/complexity trade-off. The IBO gain can be deducted by comparing the MER values of the signal with and without PAPR reduction. A target value of MER above $34 \mathrm{~dB}$ is considered. Similarly, MER gain has been deducted in a similar manner with a target value of $6.4 \mathrm{~dB}$ IBO. The IBO gain at targeted MER for ICMP with different $S$ has been summarized in Table V.

The CCDF of PAPR is not plotted as it has already been shown in [28] and [29] that variation in $S$ has very minor impact on the CCDF performance, which is a direct implication of the new kernel design. Actually, ICMP optimizes the phase correction applied to the kernel to reduce the $S$ highest peaks. As stated earlier, the CCDF of PAPR reflects on statistics of the highest peak and is not seriously influenced by the additional peaks being reduced. On the contrary to $\mathrm{CCDF}$, as shown in Fig. 14 giving the MER results versus the IBO, parameter $S$ impacts much more the performance of ICMP.

\section{B. GICMP Algorithm Optimization}

Since, GICMP is supposed to deal with the latency problem that arises in higher modes, the $32 \mathrm{~K}$ mode is considered for simulation analysis by fixing $S=100$. 32K mode is chosen as it is to the best of our knowledge, the preferred mode for the deployment of the terrestrial broadcast.

1) Optimization of the Number of Groups: GICMP with different set of groups $G=\{1,2,8\}$ has been simulated and the MER results are plotted in Fig. 15. GICMP even with only one group (i.e., $G=1$ ) outperforms the classical TTPC scheme suggested in the DVB-T2 standard, with 30 iterations, both in terms of latency and IBO gain by $0.2 \mathrm{~dB}$ at $34 \mathrm{~dB}$ of MER. This translates into a huge reduction of processing delay for GICMP w.r.t. TTPC, since the peak detection process is one of the longest. As the $G$ size increases, we can notice that GICMP offers more IBO gain as shown in Table VI.

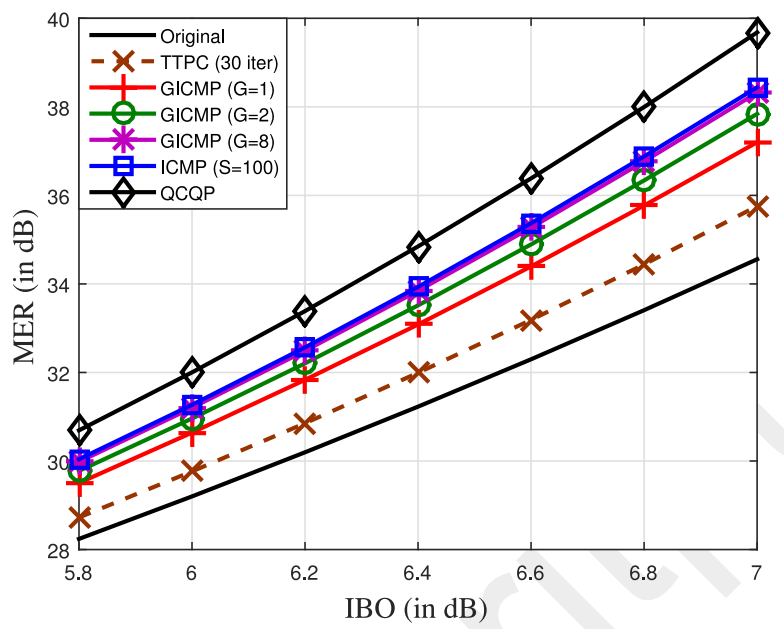

Fig. 15. MER with GICMP, $S=100$ for DVB-T2 with 64 QAM in $32 \mathrm{~K}$ mode with Rapp model HPA, $p=10$.

TABLE VI

Relative GAINS FOR GICMP W.R.T. THE ORIGINAL SignAL AND LOSS W.R.T. QCQP FOR RAPP MODEL HPA, $p=10$, IN 32K MOdE

\begin{tabular}{|c|c|c|c|}
\hline$G$ & $\begin{array}{c}\text { IBO gain w.r.t. } \\
\text { original signal }^{\dagger}\end{array}$ & $\begin{array}{c}\text { MER gain w.r.t. } \\
\text { original signal }^{\star}\end{array}$ & $\begin{array}{c}\text { MER loss w.r.t. } \\
\text { signal with QCQP }^{\ddagger}\end{array}$ \\
\hline 1 & $0.363 \mathrm{~dB}$ & $1.85 \mathrm{~dB}$ & $1.77 \mathrm{~dB}$ \\
\hline 2 & $0.433 \mathrm{~dB}$ & $2.29 \mathrm{~dB}$ & $1.33 \mathrm{~dB}$ \\
\hline 8 & $0.481 \mathrm{~dB}$ & $2.61 \mathrm{~dB}$ & $1.01 \mathrm{~dB}$ \\
\hline
\end{tabular}

$t:$ The original signal has IBO of $6.903 \mathrm{~dB}$ at $34 \mathrm{~dB}$ of MER

$\star$ : The original signal has MER of $31.23 \mathrm{~dB}$ at $6.4 \mathrm{~dB}$ of IBO

‡: The signal with QCQP has MER of $34.85 \mathrm{~dB}$ at $6.4 \mathrm{~dB}$ of IBO

Interestingly, it is worth noting that GICMP with $G=8$ has almost the same performance as ICMP with an additional IBO gain of almost $0.3 \mathrm{~dB}$ compared to the DVB-T2 TR algorithm. These results show that the proposed PAPR reduction technique offers a very good complexity/performance/latency trade-off, for the implementation in future DVB-T2 and ATSC 3.0 transmitters. One more advantage of ICMP and GICMP compared to DVB-T2 algorithm is that the research of peaks is done at nominal frequency without requiring oversampling by a factor of 4, as required for DVB-T2 TR algorithm.

2) Impact of the Power Control Level: In order to understand the impact of PC level on GICMP, simulation has been done by fixing GICMP parameters $S=100$ and $G=8$. For different PC levels $\lambda=\{3,5,10\} \mathrm{dB}$ the CCDF and MER are shown in Fig. 16 and Fig. 17 respectively. Owing to the very high complexity, the CCDF of PAPR of signal with QCQP in $32 \mathrm{~K}$ mode has not been plotted. As expected, high $\lambda$ hampers the PAPR reduction performance of GICMP.

The CCDF of PAPR at $10^{-3}$ value with GICMP for different $\lambda$ values are summarized in Table VII. At $10^{-3}$ value, the effective PAPR gain w.r.t. the original signal is $\{0.74,1.27,1.56\} \mathrm{dB}$ respectively for $\lambda=\{3,5,10\} \mathrm{dB}$. MER comparison of GICMP and QCQP with different $\lambda$ values is summarized in Table VIII.

3) Complexity Aspects: The computational complexity of finding the $S$ highest peaks is $\mathcal{O}(N)$, which is of the same order of complexity as that of TTPC. This is much less than the computational complexity of QCQP, which is $\mathcal{O}\left(R N^{2}\right)$. 


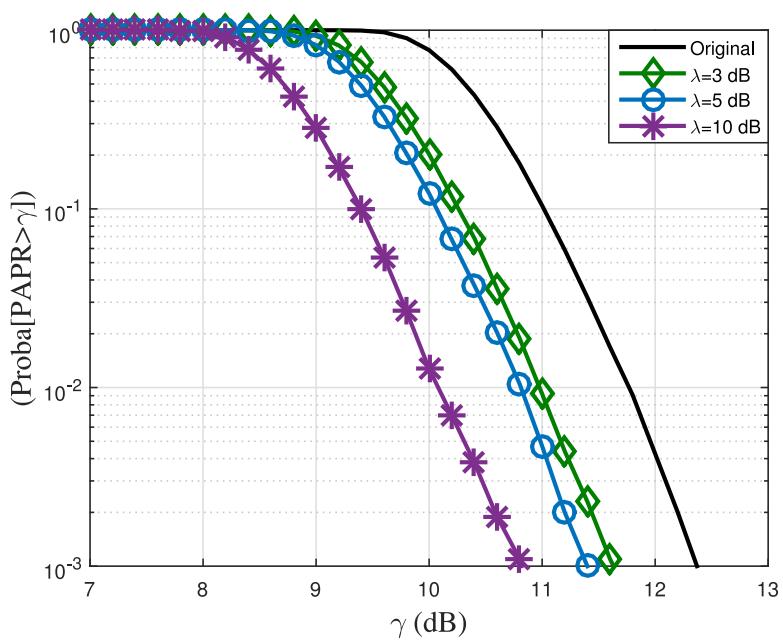

Fig. 16. CCDF of PAPR with GICMP, $S=100, G=8$ for DVB-T2 with $64 \mathrm{QAM}$ in $32 \mathrm{~K}$ mode for different $\lambda$ values.

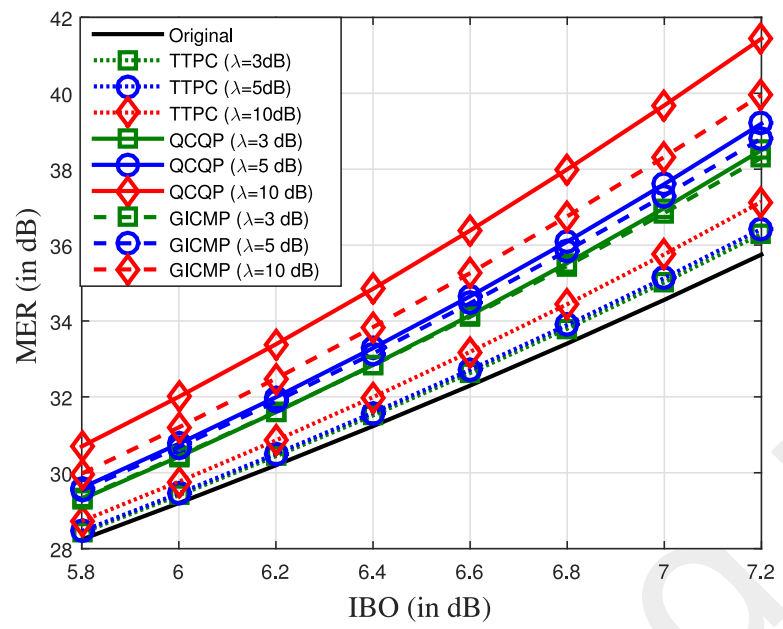

Fig. 17. MER with TTPC, QCQP, GICMP, $S=100, G=8$ for DVB-T2 with 64 QAM in $32 \mathrm{~K}$ mode with Rapp model HPA, $p=10$ for different $\lambda$ values.

TABLE VII

CCDF OF PAPR AT $10^{-3}$ VALUE FOR GICMP FOR DIFFERENT $\lambda$, IN $32 \mathrm{~K}$ MODE

\begin{tabular}{|c|c|}
\hline$\lambda$ & CCDF OF PAPR AT $10^{-3}$ \\
\hline 3 & $11.63 \mathrm{~dB}$ \\
\hline 5 & $11.10 \mathrm{~dB}$ \\
\hline 10 & $10.81 \mathrm{~dB}$ \\
\hline
\end{tabular}

TABLE VIII

COMPARISON OF MER FOR GICMP AND QCQP AND RELATIVE LOSS W.R.T. QCQP WITH DIFFERENT $\lambda$ VALUES FOR RAPP MODEL HPA, $p=10$ IN 32K MODE

\begin{tabular}{|c|c|c|c|}
\hline \multirow{2}{*}{$\lambda$} & \multicolumn{2}{|c|}{ MER at 6.4 dB of IBO } & Relative MER loss \\
\cline { 2 - 3 } & QCQP & GICMP & w.r.t. QCQP \\
\hline 3 & $32.84 \mathrm{~dB}$ & $32.83 \mathrm{~dB}$ & $0.01 \mathrm{~dB}$ \\
\hline 5 & $33.29 \mathrm{~dB}$ & $33.14 \mathrm{~dB}$ & $0.15 \mathrm{~dB}$ \\
\hline 10 & $34.85 \mathrm{~dB}$ & $33.84 \mathrm{~dB}$ & $1.01 \mathrm{~dB}$ \\
\hline
\end{tabular}

The DVB-T2 TR algorithm needs real-time kernel generation and also memory for its storage. Also, at the end of each iteration the power of PRTs needs to be checked so that PC constraint is respected. This hampers efficient usage of PRT
TABLE IX

COMPARISON ANALYSIS OF DifFERENT TR ALgORITHMS FOR DVB-T2 AND ATSC 3.0

\begin{tabular}{|c|c|c|c|c|}
\hline Criteria & DVB-T2 TR & QCQP & ICMP & GICMP \\
\hline Performance & $\begin{array}{c}\text { Non-optimal } \\
\star\end{array}$ & $\begin{array}{l}\text { Optimal } \\
\star \star \star \star\end{array}$ & $\begin{array}{c}\text { Quasi- } \\
\text { optimal } \\
\star \star \star\end{array}$ & $\begin{array}{c}\text { Quasi- } \\
\text { optimal } \\
\star \star \star\end{array}$ \\
\hline Complexity & $\begin{array}{l}\text { Low } \\
\mathcal{O}(N) \\
\star \star \star\end{array}$ & $\begin{array}{c}\text { Very high } \\
\mathcal{O}\left(R N^{2}\right) \\
\star\end{array}$ & $\begin{array}{l}\text { Low } \\
\mathcal{O}(N) \\
\star \star \star\end{array}$ & $\begin{array}{c}\text { Low } \\
\mathcal{O}(N) \\
\star \star \star \star\end{array}$ \\
\hline Latency & $\begin{array}{l}\text { Low } \\
\star \star \star\end{array}$ & $\begin{array}{c}\text { Very high } \\
\star\end{array}$ & $\begin{array}{l}\text { High } \\
\star \star\end{array}$ & $\begin{array}{l}\text { Low } \\
\star \star \star \star\end{array}$ \\
\hline $\begin{array}{l}\text { Real-time kernel } \\
\text { generation }\end{array}$ & $\begin{array}{c}\text { Yes } \\
\star\end{array}$ & $\begin{array}{c}\text { No } \\
\star \star \star \star\end{array}$ & $\begin{array}{l}\text { Yes }^{\dagger} \\
\star \star \star\end{array}$ & $\begin{array}{l}\text { Yes }^{\dagger} \\
\star \star \star\end{array}$ \\
\hline $\begin{array}{l}\text { Memory to } \\
\text { store kernel }\end{array}$ & $\begin{array}{c}\text { Yes } \\
\star\end{array}$ & $\begin{array}{c}\text { No } \\
\star \star \star \star\end{array}$ & $\begin{array}{c}\text { No } \\
\star \star \star \star\end{array}$ & $\begin{array}{c}\text { No } \\
\star \star \star \star\end{array}$ \\
\hline $\begin{array}{l}\text { PC check } \\
\text { requirement }\end{array}$ & $\begin{array}{c}\text { Yes } \\
\star\end{array}$ & $\begin{array}{c}\text { Yes } \\
\star\end{array}$ & $\begin{array}{c}\text { No } \\
\star \star \star \star\end{array}$ & $\begin{array}{c}\text { No } \\
\star \star \star \star\end{array}$ \\
\hline $\begin{array}{l}\text { Efficient use } \\
\text { of PRT power }\end{array}$ & $\begin{array}{c}\text { No } \\
\star\end{array}$ & $\begin{array}{c}\text { Yes } \\
\star \star \star \star\end{array}$ & $\begin{array}{c}\text { Yes } \\
\star \star \star \star\end{array}$ & $\begin{array}{c}\text { Yes } \\
\star \star \star \star\end{array}$ \\
\hline $\begin{array}{l}\text { Compatibility } \\
\text { with standard }\end{array}$ & $\begin{array}{c}\text { Yes } \\
\star \star \star \star\end{array}$ & $\begin{array}{c}\text { Yes } \\
\star \star \star \star\end{array}$ & $\begin{array}{c}\text { Yes } \\
\star \star \star \star\end{array}$ & $\begin{array}{c}\text { Yes } \\
\star \star \star \star\end{array}$ \\
\hline
\end{tabular}

$\dagger$ : Can be done with simple phase shift operations

power. Whereas, in the proposed algorithms the kernel generation can be done with simple phase shift operations and no PC check is required because PRT power is kept constant (i.e., equal to $P_{\max }$ ).

4) Quasi-Optimality: From Table VIII, it can be noticed that unlike TTPC, the GICMP algorithm with $G=8$ achieves quasi-optimal performance at low values of $\lambda$, while gaining heavily on complexity and latency. As summarized in Table VIII, the relatives MER loss w.r.t. QCQP for $\lambda=$ $\{3,5,10\} \mathrm{dB}$ are $\{0.01,0.15,1.01\} \mathrm{dB}$ respectively. It is very interesting to note that the relative MER loss and $\eta$ are very meager for GICMP, for $\lambda=\{3,5\} \mathrm{dB}$, where PRT power allocation is much lower. Thus, achieving a quasi-optimal performance with a complexity of $\mathcal{O}(N)$ with low latency is very exciting result. This is due to the fact that, the new kernel is not a Dirac pulse as used in the DVB-T2 TR algorithm, but a comb-like one. This fundamental aspect is the reason of the quasi-optimal performance offered by the proposed algorithms.

Even though, our main criteria are performance, complexity and latency, a comparative analysis with some additional ones are presented in Table IX. As per these criteria, GICMP seems to offer the best performance/complexity/latency trade-off.

5) Latency Aspects: In classical ICMP, the number of iterations is equal to the total number of reserved subcarriers, while in GICMP it is equal to the number of groups. This explains the substantial decrease in the implementation latency. In ICMP, at the end of each iteration, a peak search operation and then a peak reduction is carried out and the resulting signal is the input data signal for the next iteration. In GICMP, due to concurrency involved in the implementation, the original data signal remains the same for all groups and the final peak cancellation signal is computed by adding the peak cancellation sub-signals from each parallel process. The number of peak search operations and kernels that can be generated in parallel for ICMP and various GICMP configurations in $32 \mathrm{~K}$ mode has been summarized in Table X. In the same Table, 
TABLE X

COMPARISON OF ICMP AND VARIOUS GICMP CONFIGURATIONS IN 32K MODE

\begin{tabular}{|c|c|c|c|}
\hline$G$ & $\begin{array}{c}\text { PRTs } \\
\text { per group }\end{array}$ & $\begin{array}{c}\text { Peak search } \\
\text { operations required }\end{array}$ & $\begin{array}{c}\text { Kernels that can be } \\
\text { generated in parallel }\end{array}$ \\
\hline 1 & 288 & 1 & 288 \\
\hline 2 & 144 & 2 & 144 \\
\hline 4 & 72 & 4 & 72 \\
\hline 8 & 36 & 8 & 36 \\
\hline 16 & 18 & 16 & 18 \\
\hline 32 & 9 & 32 & 9 \\
\hline $288^{\dagger}$ & 1 & 288 & 1 \\
\hline
\end{tabular}

$\uparrow$ No Grouping i.e. ICMP.

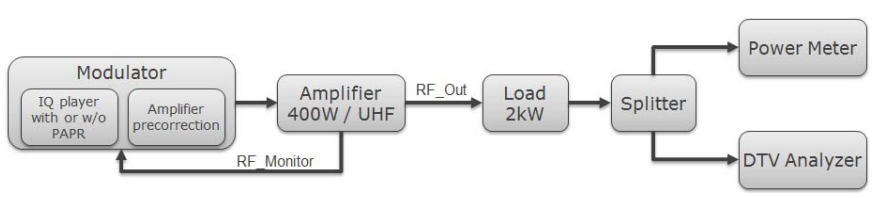

Fig. 18. Diagram of the test bench.

we can see the number of kernels that have to be generated in parallel. Ideally, in $32 \mathrm{~K}$ mode, GICMP with $G=288$ is equivalent to ICMP, which needs 288 peak search operations inducing huge processing delay. With sufficiently large number of groups, GICMP can achieve the performance of ICMP with very low latency and reasonable complexity. For FPGA or ASIC implementation, the choice of $G$ between 8 and 16 is allowed.

From the measurement results obtained from the experimental platform, we have made some conclusion regarding the proposed GICMP technique. The GICMP algorithm with $G=8$ can be implemented using a Xilinx Kintex7 family (XC7K160T) FPGA with the parallel architecture. It has been shown that 263,560 processor clock cycles are required for GICMP with $G=8$. This is the equivalent of 8.04 times the duration of an OFDM symbol, this falls within the 8 to 10 times acceptable delay limit [9]. In DVB-T2, the duration of one OFDM symbol in $32 \mathrm{~K}$ mode including shortest guard interval is $3612 \mu \mathrm{s}$.

\section{Testbed Platform EXPERIMENTS AND MEASUREMENTS}

This section presents the performance measurements for the candidate algorithms using real equipments. The platform includes a real-time signal generator with real-time self-adaptive pre-distortion and real power amplifiers. The goal is to evaluate the impact of the PAPR reduction methods on the system as a whole.

The first series of tests, measure the MER performance and the impact on pre-distortion convergence of various candidates. The second series of tests, focus on power related metrics such as power consumption and efficiency.

\section{A. Platform Presentation}

The test bench is described in Fig. 18. This test bench is able to follow MER variation over time and thus to analyze algorithm convergence and stability. As per the DVB-T2

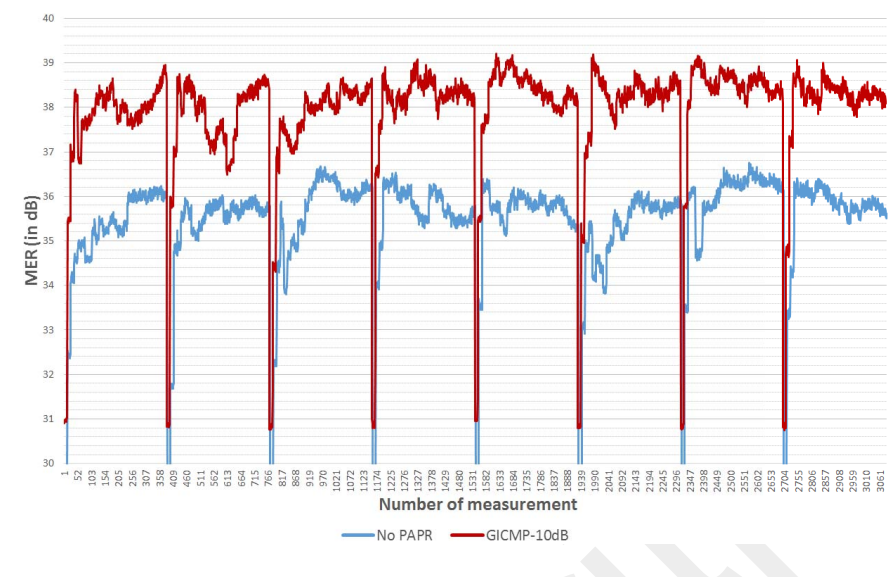

Fig. 19. MER vs time measurements without PAPR reduction and with GICMP, for $\lambda=10 \mathrm{~dB}$ and class AB amplifier at output power $400 \mathrm{~W}$.

specification, multiple I/Q sample data are generated with the following common characteristics

- FFT size: $32 \mathrm{~K}$

- Channel width: $8 \mathrm{MHz}$

- Guard Interval: 1/128

- Data constellation: 64 QAM

- Pilot Pattern: PP7

The signal generator is implemented in a compact module, which is integrated with the digital pre-correction. Once convergence is completed, then all performance measurements (power, MER, shoulders, etc.) are made.

The output of the modulator is fed to an amplifier. Two amplifiers are used during the tests:

- a Class AB amplifier, delivering a $400 \mathrm{~W}$ output power in nominal conditions over the whole UHF band, runs with a $5 \mathrm{dBm}$ input level and used at $674 \mathrm{MHz}$. Furthermore, the operating point of the class $\mathrm{AB}$ amplifier corresponds to an IBO around $6.9 \mathrm{~dB}$, to deliver the nominal output power of $400 \mathrm{~W}$.

- a Doherty amplifier, delivering a $210 \mathrm{~W}$ output power in nominal conditions, runs with a $0 \mathrm{dBm}$ input level and used at $642 \mathrm{MHz}$. Similarly, the operating point of the Doherty amplifier corresponds to an IBO around $7.1 \mathrm{~dB}$, to deliver the nominal output power of $210 \mathrm{~W}$.

The output of the amplifier is connected to an attenuator. The power is then monitored with a power meter, directly providing measurements in Watts.

A monitoring output is split into two paths. One is fed to the modulator as a feedback input used by the pre-distortion algorithm, the other one is fed to a TV analyzer providing spectrum, MER and shoulder measurements.

\section{B. Impact of PAPR Reduction}

The parameters of the PAPR reduction algorithm are the following

- The PC level, $\lambda=10 \mathrm{~dB}$,

- The number of detected peaks at each iteration, $S=100$,

- The PAPR reduction algorithm is GICMP with $G=8$ groups (i.e., 36 PRTs per group). 
TABLE XI

Tests Results With THE GICMP AlgorithM

\begin{tabular}{|c|c|}
\hline ALGORITHM & AVERAGE MER (in dB) \\
\hline No PAPR reduction & 35.9 \\
\hline GICMP & 38.4 \\
\hline
\end{tabular}

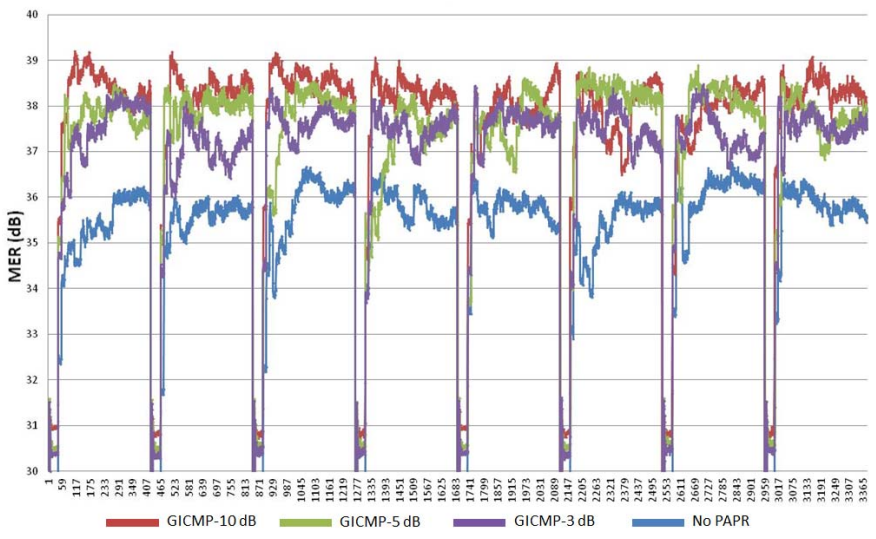

Fig. 20. MER vs time measurements without PAPR reduction and with the GICMP algorithm with Class AB test amplifier at output power $400 \mathrm{~W}$.

The MER performance for the original signal without PAPR reduction and with the GICMP algorithm in the case of Class $\mathrm{AB}$ amplifier, and for the same IBO, are displayed over time in Fig. 19 respectively. In that figure, the MER measured during eight DVB-T2 frames is plotted. During a given DVB-T2 frame duration, the average MER measured with and without PAPR reduction has been summarized in Table XI. It can be concluded that the proposed GICMP algorithm has very good PAPR reduction performance with $2.5 \mathrm{~dB}$ gain in MER.

This MER gain equal to $2.5 \mathrm{~dB}$ measured on the testbed is slightly lower than the MER gain obtained by simulation with GICMP with the same parameters.

In our analysis, a trade-off among the three parameters can be achieved. They are MER, output power $P_{\text {out }}$ and power gain. Section V-C highlights the impact of PC on MER and Section V-D deals with impact of PAPR reduction on power gain. Likewise, Section V-E showcases that power consumption, i.e., the DC power of the HPA, can be reduced with PAPR reduction for a target MER at constant output RF power.

\section{Impact of Power Control Level}

In order to evaluate the MER performance with $\lambda=\{3,5,10\}$ $\mathrm{dB}$, we have performed tests with both Class $\mathrm{AB}$ and Doherty amplifiers. The MER vs time measurements have been plotted for the same IBO in Fig. 20 and Fig. 21, respectively. For both amplifiers and various power constraint values, the algorithm is stable (i.e., the variation during one frame is inferior to $1 \mathrm{~dB}$ ) after convergence. It has to be noted that the total transmit power is the same for all the $\lambda$ values.

According to PC constraint in DVB-T2, the GICMP algorithm introduces a power increase on reserved subcarriers compared to data subcarriers. However, in order to compare in fair conditions, the power of the data subcarriers is slightly decreased, in order to get the same total power for the original

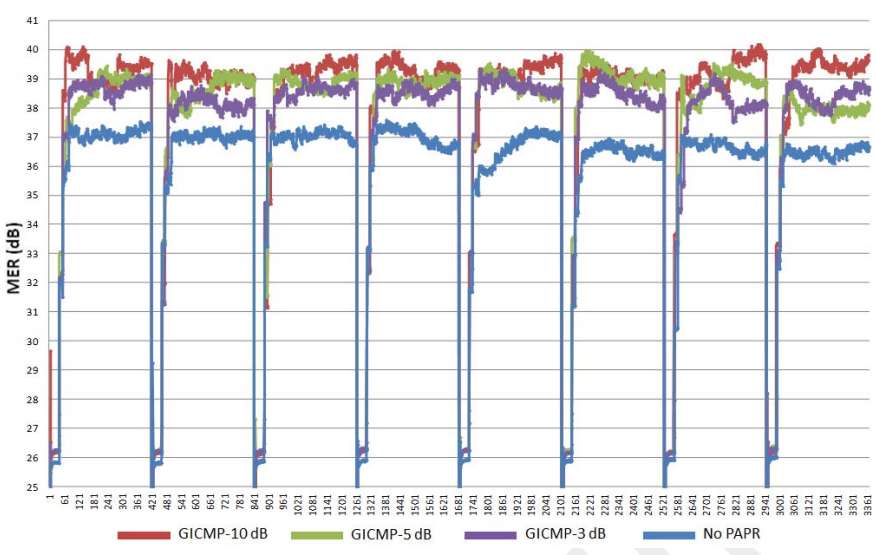

Fig. 21. MER vs time measurements without PAPR reduction and with the GICMP algorithm with Doherty test amplifier at output power $210 \mathrm{~W}$.

TABLE XII

AVERAGE MER vs $\lambda$

\begin{tabular}{|c|c|c|}
\hline & CLASS AB MER & DOHERTY MER \\
\hline No PAPR reduction & $35.9 \mathrm{~dB}$ & $37.0 \mathrm{~dB}$ \\
\hline GICMP, $\lambda=3 \mathrm{~dB}$ & $37.5 \mathrm{~dB}$ & $38.6 \mathrm{~dB}$ \\
\hline GICMP, $\lambda=5 \mathrm{~dB}$ & $38.0 \mathrm{~dB}$ & $39.0 \mathrm{~dB}$ \\
\hline GICMP, $\lambda=10 \mathrm{~dB}$ & $38.2 \mathrm{~dB}$ & $39.2 \mathrm{~dB}$ \\
\hline
\end{tabular}

signal and for the one with GICMP PAPR reduction. As mentioned previously, the increase of PRT power with the same total output power, leads to a receiver sensitivity loss. So, sensitivity loss can be minimized, by lowering $\lambda$, thus minimizing the impact of the PAPR reduction mechanism on the overall mean power level. Additional tests are performed with a power constraint of $5 \mathrm{~dB}$ and $3 \mathrm{~dB}$, in order to evaluate the impact on performance with these sub-optimal configurations. The average MER is displayed in Table XII. The best performance is reached for $\lambda=10 \mathrm{~dB}$ leading to a MER gain, compared to the original signal, of $2.3 \mathrm{~dB}$ for the class $\mathrm{AB}$ and $2.2 \mathrm{~dB}$ for the Doherty amplifier for an IBO around $7 \mathrm{~dB}$.

Furthermore, we can say that the 2.3 to $2.5 \mathrm{~dB}$ MER gain observed for an IBO around $7 \mathrm{~dB}$ can also be obtained for a lower IBO in order to improve the MER from roughly $32 \mathrm{~dB}$ to $34 \mathrm{~dB}$.

\section{Impact on Power Gain}

The gain we had at MER level can be translated into an output power gain for a given target MER, i.e., fairly constant quality of signal. This has been experienced in our testbed by modifying the output power, starting with $P_{\text {out }}=100 \mathrm{~W}$ without PAPR reduction and then increasing such power level until reaching MER of $36 \mathrm{~dB}$ with the use of GICMP. For these additional measurements the parameter $\lambda$ has been fixed to $5 \mathrm{~dB}$ in order to keep the receiver sensitivity loss $\eta$ below $0.1 \mathrm{~dB}$ (see Table III). It has to be noted that increasing $P_{\text {out }}$ is simply obtained by reducing the IBO. This test shows a $10 \%$ gain of output power, when GICMP is used, as depicted in Table XIII. This gain would have been much weaker with the state of art DVB-T2 TR algorithm, as evident from simulation results. So, this is significant because high power output 
TABLE XIII

OUtPut POWER AT CONSTANT MER

\begin{tabular}{|c|c|c|}
\hline & NO PAPR REDUCTION & GICMP, $\lambda=\mathbf{5} \mathbf{d B}$ \\
\hline$P_{\text {out }}$ & $100 \mathrm{~W}$ & $110 \mathrm{~W}$ \\
\hline
\end{tabular}

TABLE XIV

MER Gain And Shoulder Gain at Constant Power Level

\begin{tabular}{|c|c|c|}
\hline & NO PAPR REDUCTION & GICMP, $\lambda=\mathbf{5}$ dB \\
\hline Power consumption & $313 \mathrm{~W}$ & $284 \mathrm{~W}$ \\
\hline
\end{tabular}

with broadcasting systems is usually obtained by using in parallel several amplifiers. The resulting output power gain of the global chain is then also around $10 \%$, which is already substantial considering several $\mathrm{kW}$ transmitters.

\section{E. Impact of Power Consumption}

On the other hand we can also modify the power consumption to achieve a target MER at constant output power in order to modify its response. This is obtained by reducing the power supply of the HPA when PAPR reduction is activated and by adapting the IBO accordingly in order to obtain the same $P_{\text {out }}$ as when the PAPR reduction is not activated. Another class $\mathrm{AB}$ amplifier has been used for the measurements so that the power supply could be easily managed.

As shown by the results given in Table XIV with $P_{\text {out }}=$ $100 \mathrm{~W}$, the energy consumption can be reduced by $10 \%$. This means that by applying the GICMP algorithm to a transmitter with a basic efficiency of $32 \%$ can have its efficiency boosted to about $35.2 \%$. This reduction in energy consumption confirms the "green" benefit of the proposed algorithm at a time when all transmitter manufacturers are working at improving the energy efficiency of their systems.

\section{CONCLUSION}

It is inevitable to have PAPR reduction in DVB-T2 and ATSC 3.0 transmission systems in order to improve their HPA efficiency. In this paper, two TR-based PAPR reduction methods, namely, ICMP and GICMP were discussed that are based on a novel kernel definition. The proposed methods are based on this new simple kernel signal and an optimized computation of its phase correction. Each kernel signal is defined to deal with the reduction of multiple peaks at a time and the new TR technique leads to a better usage of the available power of the PRTs. These methods are evaluated in terms of latency, complexity and performance trade-off. Simulation results have shown that GICMP meets all the criteria. The ICMP and GICMP algorithms outperform the TR algorithm proposed in the DVB-T2 standard. Furthermore, the GICMP algorithm offers quasi-optimal performance closed to the performance of the optimal QCQP algorithm with a very reasonable complexity and low latency. The reason for the quasi-optimal performance offered by GICMP is due to the fact that the new kernel is a comb-like pulse. A testbed implementation has been used to perform an in-depth analysis of GICMP and it was found that GICMP with $S=100$ and $G=8$ has very good performance/complexity/latency trade-off. This confirms its high potential in low-latency PAPR reduction and its compatibility with existing DVB-T2 and ATSC 3.0 standards.

From the real testbed implementation, we have noticed two outcomes. Firstly, choosing $\lambda=5 \mathrm{~dB}$ instead of $10 \mathrm{~dB}$ as allowed by the DVB-T2 standard is a good trade-off. This is due to the fact that it has a minimal impact on the output signal power while preserving a MER gain. Secondly, it has been demonstrated that the proposed GICMP technique yields a MER gain of around $2 \mathrm{~dB}$ to $2.4 \mathrm{~dB}$ when used with a class $\mathrm{AB}$ and Doherty amplifier, which are the two most common types of amplifiers. At a constant MER, the benefits of the GICMP technique can either be turned into a gain of output power or a reduction of energy consumption at constant output power. In that perspective, it was further shown that gain in terms of output power or in power consumption of about $10 \%$ have been observed.

\section{ACKNOWLEDGMENT}

The authors would like to thank the engineers of Kenta for the measurements of energy consumption.

\section{REFERENCES}

[1] S. H. Han and J. H. Lee, "An overview of peak-to-average power ratio reduction techniques for multicarrier transmission," IEEE Trans. Wireless Commun., vol. 12, no. 2, pp. 56-65, Apr. 2005.

[2] M. J. F.-G. Garcia, O. Edfors, and J. M. Pacz-Borallo, "Peak power reduction for OFDM systems with orthogonal pilot sequences," IEEE Trans. Wireless Commun., vol. 5, no. 1, pp. 47-51, Jan. 2006.

[3] X. Li and L. J. Cimini, "Effects of clipping and filtering on the performance of OFDM,' IEEE Commun. Lett., vol. 2, no. 5, pp. 131-133, May 1998.

[4] T. A. Wilkinson and A. E. Jones, "Minimisation of the peak-to-mean envelope power ratio of multi-carrier transmission schemes by block coding," in Proc. IEEE VTC, vol. 2. Chicago, IL, USA, Jul. 1995, pp. $825-829$.

[5] S. H. Muller and J. B. Huber, "OFDM with reduced peak-to-average power ratio by optimum combination of partial transmit sequences," IEEE Electron. Lett., vol. 33, no. 5, pp. 368-369, Feb. 1997.

[6] R. W. Bauml, R. F. H. Fischer, and J. B. Huber, "Reducing the peak-toaverage power ratio of multi-carrier modulation by selected mapping," IEEE Electron. Lett., vol. 32, no. 22, pp. 2056-2057, Oct. 1996.

[7] B. S. Krongold and D. L. Jones, "PAR reduction in OFDM via active constellation extension," IEEE Trans. Broadcast., vol. 49, no. 3, pp. 258-268, Sep. 2003.

[8] J. Tellado, "Peak to average ratio reduction for multi-carrier modulation," Ph.D. dissertation, Dept. Elect. Eng., Stanford Univ., Stanford, CA, USA, 1999.

[9] Frame Structure Channel Coding and Modulation for a Second Generation Digital Terrestrial Television Broadcasting System (DVB-T2), ETSI Standard EN 302755 V1.1.1, Sep. 2009.

[10] ATSC Proposed Standard: Physical Layer Protocol, document S32230r56, Adv. Television Syst. Committee, Washington, DC, USA, 2016.

[11] Jornada Innovación Sistemática, EGATEL, Ourense, Spain, May 2013, p. 14.

[12] M. Mroué, A. Nafkha, J. Palicot, B. Gavalda, and N. Dagorne, "Performance and implementation evaluation of TR PAPR reduction methods for DVB-T2," Int. J. Digit. Multimedia Broadcast., vol. 2010, p. 10, Aug. 2010. [Online]. https://www.hindawi.com/journals/ijdmb/ 2010/797393/

[13] H. Li, T. Jiang, and Y. Zhou, "An improved tone reservation scheme with fast convergence for PAPR reduction in OFDM systems," IEEE Trans. Broadcast., vol. 57, no. 4, pp. 902-906, Dec. 2011.

[14] T. Jiang, C. Ni, C. Xu, and Q. Qi, "Curve fitting based tone reservation method with low complexity for PAPR reduction in OFDM systems," IEEE Commun. Lett., vol. 18, no. 5, pp. 805-808, May 2014.

[15] S. Rosati, E. A. Candreva, and G. E. Corazza, "Rotation invariant subcarrier mapping over DVB-T2 reserved tones," in Proc. IEEE Veh. Technol. Conf. (VTC Spring), Dresden, Germany, Jun. 2013, pp. 1-5. 
[16] T. Jiang, C. Ni, C. Ye, Y. Wu, and K. Luo, "A novel multi-block tone reservation scheme for PAPR reduction in OQAM-OFDM systems," IEEE Trans. Broadcast., vol. 61, no. 4, pp. 717-722, Dec. 2015.

[17] F. Tosato, M. Sandell, and M. Tanahashi, "Tone reservation for PAPR reduction: An optimal approach through sphere encoding," in Proc. IEEE Int. Conf. Commun., Kuala Lumpur, Malaysia, May 2017, pp. 1-6.

[18] C. Rapp, "Effects of HPA nonlinearity on 4-DPSK-OFDM signal for digital sound broadcasting systems," in Proc. Eur. Conf. Satellite Commun., Oct. 1991, pp. 179-184.

[19] H. E. Rowe, "Memoryless nonlinearities with Gaussian inputs: Elementary results," Bell Syst. Tech. J., vol. 61, no. 7, pp. 1519-1526, Sep. 1982.

[20] R. Van Nee and R. Prasad, OFDM for Wireless Multimedia Communications. Boston, MA, USA: Artech House, 2000.

[21] "Digital video broadcasting (DVB); measurement guidelines for DVB systems," ETSI, Sophia Antipolis, France, Rep. TR 101290 V1.2.1, May 2001.

[22] Y. Louët and J. Palicot, "A classification of methods for efficient power amplification of signals," Ann. Telecommun., vol. 63, nos. 7-8, pp. 351-368, Aug. 2008.

[23] J. Tellado and J. Cioffi, "Peak power reduction for multicarrier transmission," in Proc. IEEE CTMC GLOBECOM, Sydney NSW, Australia, Nov. 1998, pp. 219-224.

[24] M. Grant, S. Boyd, and Y. Ye. CVX-MATLAB Software for Disciplined Convex Programming. [Online]. Available: http://www.stanford.edu/boyd/cvx/

[25] "Boundary coverage assessment of digital terrestrial television broadcasting signals," ITU, Geneva, Switzerland, ITU-Recommendation BT.2143-1, p. 3, Nov. 2009.

[26] MER and Coverage in Broadcast Network Planning, v.01.01, Rohde \& Schwarz, Munich, Germany, pp. 3-7, Oct. 2013.

[27] Digital Video Broadcasting (DVB); Frame Structure Channel Coding and Modulation for a Second Generation Digital Terrestrial Television Broadcasting System (DVB-T2), ETSI Standard EN 302755 V1.3.1, Sep. 2011.

[28] R. Mounzer, M. Crussière, Y. Nasser, and J.-F. Hélard, "Tone reservation based PAPR reduction technique with individual carrier power allocation for multiple peaks reduction," in Proc. IEEE Veh. Technol. Conf. VTCSpring, Glasgow, U.K., May 2015, pp. 1-6.

[29] S. S. K. C. Bulusu, M. Crussière, J.-F. Hélard, R. Mounzer, and Y. Nasser, "A low latency algorithm for efficient PAPR reduction for DVB-T2 and ATSC 3.0 broadcast," in Proc. IEEE Int. Symp. Broadband Multimedia Syst. Broadcast., Cagliari, Italy, Jun. 2017, pp. 1-5. 